\title{
Climate at the equilibrium line of glaciers
}

\author{
ATsumu OHMURA, \\ Geographisches Institut, Eidgenössische Technische Hochschule, CH-8057 Zürich, Switzerland \\ Peter Kasser \\ im Rennweg 45, CH-8704 Herrliberg, Switzerland \\ MARTIN FUNK \\ Versuchsanstalt für Wasserbau, Hydrologie und Glaziologie, Eidgenössische Technische Hochschule, \\ CH-8092 Zürich, Switzerland
}

\begin{abstract}
The relationships between temperature, precipitation and radiation on glacier equilibrium lines are investigated, using 70 glaciers for which the mass balance and meteorological observations have been carried out for sufficiently long periods. It is found that the characteristic climate at glacier equilibrium lines can be described using the summer 3 months' temperature in a free atmosphere, annual total precipitation, and the sum of global and long-wave net radiation. All of these are measured at or very near the equilibrium-line altitudes. Then, it is shown how the shift of the equilibrium line will occur as a result of a climatic change. Finally, the effect of the shift of the equilibrium line on the annual mean specific mass balance is analytically derived and compared with observations. The present results make it possible to identify the altitudes in climate models where glacierization should begin, and to evaluate the mass-balance changes as a result of possible future changes in the climate.
\end{abstract}

\section{INTRODUCTION}

Glacier equilibrium lines are very important because they represent the lowest boundary of the climatic glacierization. The climates which prevail at glacier equilibrium lines are considered to be just sufficient to maintain the existence of glaciers. A thorough knowledge of the climates at equilibrium lines is, therefore, essential for understanding the relationship between climatic changes and glacier variations. Investigations along these lines of thought were previously pursued by Ahlmann (1948) and Loewe (1971). In view of the recent improvement in information on the equilibrium line, availability of more climatic data and the new requirements for climate modelling, the authors decided to formulate this problem from a different viewpoint.

Glacier equilibrium lines also have other important meanings. First, the year-to-year variation of the equilibrium-line altitude (ELA) is a good indicator of the variation in the total annual mass balance of the glacier. Secondly, but closely related to the first point, the largest standard deviation of annual zonal mass balance (mass balance for a certain altitude zone) is usually observed at around the ELA. Thirdly, a substantial part of the glacier meltwater originates from near the ELA (Ohmura and others, 1986).
There are, at least, two approaches to understanding the climate at the ELA. One is to pursue the processes of accumulation and melt, where the latter can be vigorously investigated from the energy-balance principle. The other approach can be made from the viewpoint of scaling, whereby the relevant variables for the ELA are selected and the relationships between them and the annual accumulation or ablation are sought statistically. The present work is aimed at finding the relationship between the climate and the glacier equilibrium line, based on the second approach. The ELA values used in this work are all directly derived from the mass-balance measurements. The glaciers considered in this work are those in mid-latitudes and polar regions. The glaciers in the tropics behave differently and will be treated separately later.

\section{PRESENT DISTRIBUTION OF THE ELA}

Despite its importance, the ELA is often treated crudely, confused with the snow line, with little reference to the mass balance. In view of this problem, it is worthwhile looking at the large-scale distribution of the ELA in relation to some climatological elements. Figure $1 \mathrm{a}, \mathrm{b}, \mathrm{c}$, $\mathrm{d}$ and e illustrate the distributions of the ELA for the 

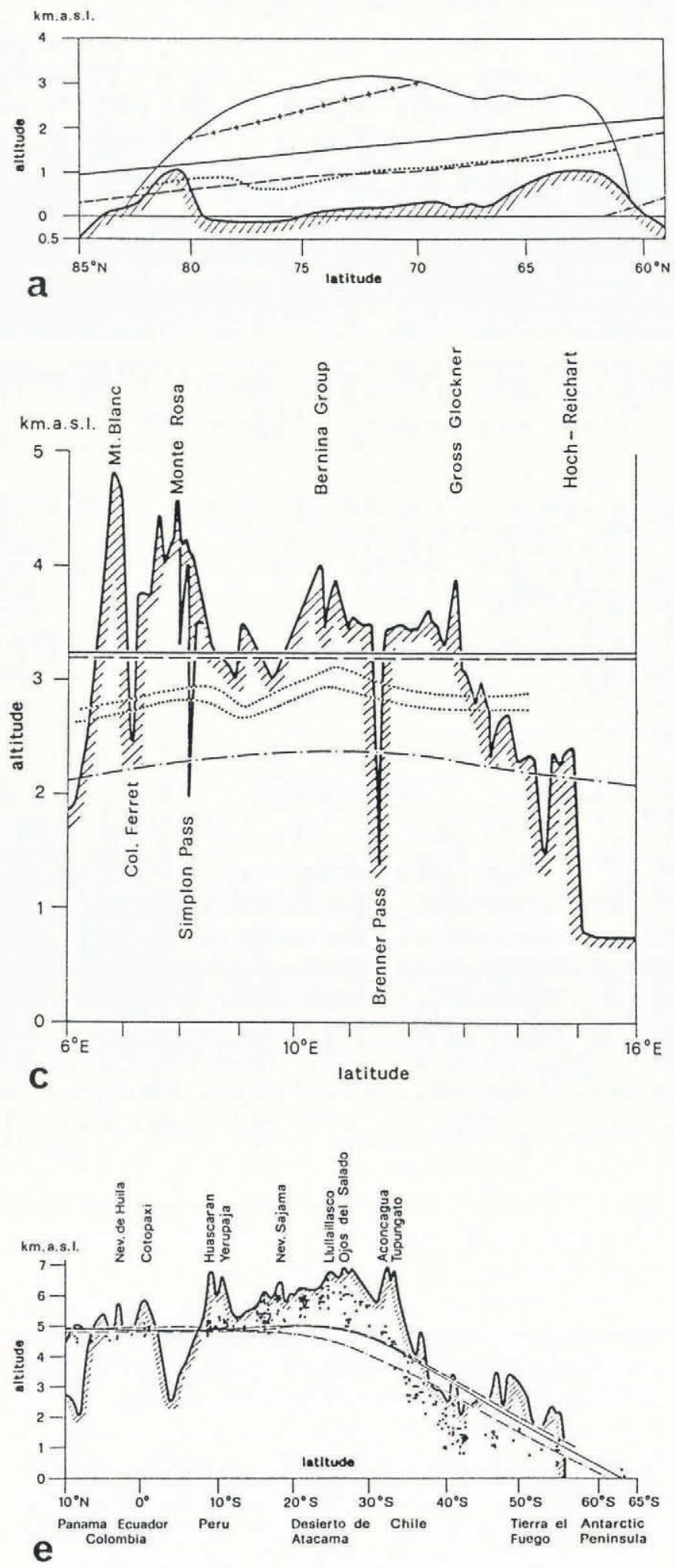

west slope of Greenland, west Scandinavia, the Alps, central Asia and the Andes, respectively. Other glacioclimatologically important lines are also plotted to assist interpretation.

The ELA on the west slope of the Greenland ice sheet (Fig. 1a) falls from about $1500 \mathrm{~m}$ a.s.l. at the southern end to $700 \mathrm{~m}$ a.s.l. on the northern slope. Often, the annual mean $0^{\circ} \mathrm{C}$ isotherm is used as the ELA (Källén and others, 1979; Oerlemans and Van der Veen, 1984). It should be noted that the $0^{\circ} \mathrm{C}$ annual mean surface temperature barely touches the southern tip of Greenland and does not even intercept the ice sheet. This fact
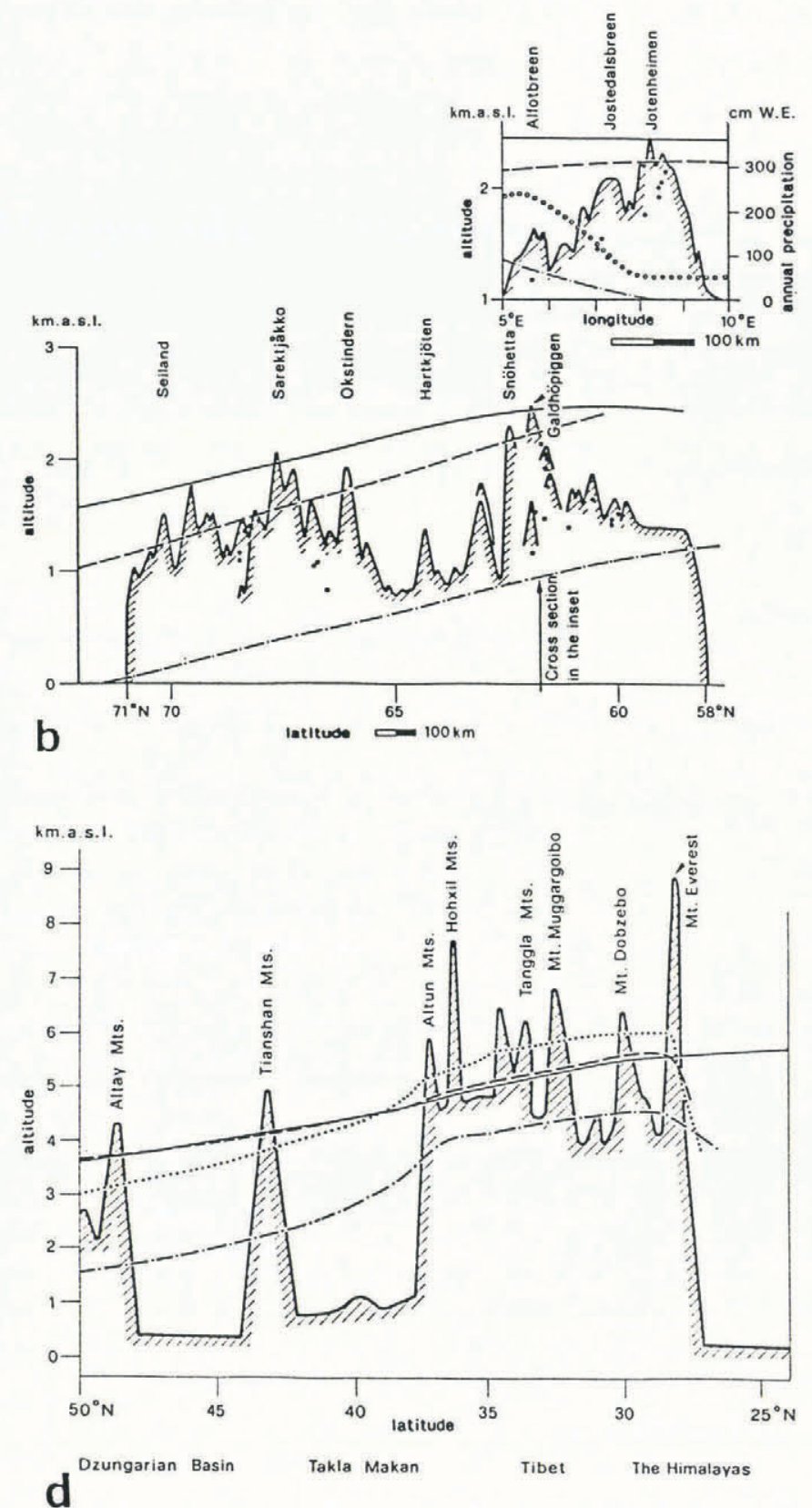

demonstrates how unrealistic it is to approximate the ELA with the $0^{\circ} \mathrm{C}$ annual mean surface temperature. If, for some reason, the $0^{\circ} \mathrm{C}$ annual mean surface temperature is preferred to be related to the ELA, the summer 3 months (JJA) surface temperature comes closest to the ELA on the west slope of Greenland. A local depression of the ELA at around $77^{\circ} \mathrm{N}$ is due to greater precipitation from the North Water, one of the major recurring polynyas in the Northern Hemisphere (Ohmura, 1976).

The ELAs are presently best known in Norway. The ELAs in Figure $1 \mathrm{~b}$ are all calculated by the long-term mass-balance measurements with wellchosen stake networks. All ELAs are distributed within the annual mean and summer temperature zero lines, descending on an average from 1400 to $1600 \mathrm{~m}$ in Folgefonni/Hardangerjöklen to $1100-1350 \mathrm{~m}$ in the Storsteinfjellbreen/Blaisen area over $900 \mathrm{~km}$. A much steeper descent in the ELA is seen, however, from the interior of the Scandinavian peninsula to the Atlantic coast. In the latitudinal cross-section along $61^{\circ} 40^{\prime} \mathrm{N}$ from Memurubreene, through Jostendalsbreen to Alfot- 
$\triangleleft$ Fig. 1. a. Distribution of equilibrium line (dots) for West Greenland. $0^{\circ} \mathrm{C}$ isothermal lines for the free atmosphere during June, July and August (solid line), for the surface during the same 3 months (broken line), and for the annual mean surface temperature (broken line and dot) are also plotted. Glaciological information is according to Schytt (1955), Braithwaite (1980), Olesen (1986), Weidick and Thomsen (1986) and personal communications from R. J. Braithwaite and H. H. Thomsen. Climatological data are according to Scherhag (1969) and Ohmura (1987). The broken line and cross represent the dry snow line by Benson (1962). b. Distribution of equilibrium line for Scandinavia. The ELAs of individual glaciers are expressed as dots. The meanings of the other lines are the same as in Figure 1a, except for the open circles in the top right inset which indicate annual precipitation with the scale on the righthand side. Glaciological data are according to Kasser (1973), Müller (1977) and Haeberli (1985). Climatological data are according to Scherhag (1969), NCAR World Weather Disc Records - Upper Air (TD-9648), WMO (1971), British Meteorological Office (1978) and Wernstedt (1985). c. Distribution of equilibrium line in the Alps. The most likely altitudes of the equilibrium lines are found within the two dotted lines. The $0^{\circ} \mathrm{C}$ isothermal lines are the same as for Figure 1a. Glaciological data are according to Hoinkes (1970), Kasser (1973), Müller (1977), Kuhn (1981), Funk (1985), Haeberli (1985), Moser and others (1986), Funk and Aellen (unpublished). Climatological data are according to Scherhag (1969) and WMO (1971). d. Distribution of equilibrium line for Central Asia. The mean altitude of the equilibrium line is shown as dots. The $0^{\circ} \mathrm{C}$ isothermal lines are the same as for Figure 1a. Glaciological data are according to Fujii and others (1976), Ageta and Satow (1978), Yasunari and Inoue (1978) and Shi (1988). Climatological data are according to NCAR World Weather Disc Records - Upper Air (TD-9648), WMO (1982) and unpublished meteorological data for Tibet, Tianshan and Altai Shan provided by the Lanzhou Institute of Glaciology and Geocryology, Academia Sinica. e. The distribution of equilibrium lines for the Andes. The ELAs of individual glaciers are expressed as dots. The $0^{\circ} \mathrm{C}$ isothermal lines are the same as for Figure 1a. The glaciological data are according to Nogami (1976), Jordan (1984) and Ames (1985). Climatological data are according to Prohaska (1976), Miller (1976), Johnson (1976), NCAR World Weather Disc Records - Upper Air (TD-9648) and U.S. Department of Commerce (1982).

breen, the ELA descends from 2050 to $1150 \mathrm{~m}$ in just over $150 \mathrm{~km}$. In the east-west cross-section of southern Scandinavia, annual precipitation decreases rapidly from over $2000 \mathrm{~mm}$ within the first $50 \mathrm{~km}$ and reaches a minimum of less than $500 \mathrm{~mm}$ at Jotenheimen. This is one of the regions in the world where the precipitation gradient from the coast to the interior shows its effect clearly on the ELAs.

In the Alps, the ELAs (Fig. 1c) tend to appear at about $700 \mathrm{~m}$ above the zero annual mean temperature. The ELAs climb slowly from the French Alps to the Swiss Alps, reaching more than $3200 \mathrm{~m}$ on the north side of the Pennine Alps, especially in the Mischabel Range. The ELAs descend in the region of the Adula Group which is open to the Mediterranean. In the Austrian Alps, the highest ELAs are observed in the Ötztal Alps.

The ELA decrease to the south on the southern slope of the Himalaya (Fig. 1d) is due to the effect of the summer monsoon, greater precipitation and lower summer temperature in comparison with the northern lee side. On the Tibetan Plateau, the ELA falls only slightly with increase in latitude. The meridional temperature gradient in the middle troposphere is the smallest over the Tibetan Plateau within the entire Northern Hemisphere. The sudden drop in the ELA in the Tien Shan and the Altay Mountains is mainly due to the increase in vapour flux transported from the Atlantic (Xiao, 1981).

In the Andes (Fig. 1e), the ELA increases from the Equator towards $25^{\circ} \mathrm{S}$ which is at the latitude equivalent to the Atacama Desert on the Chilean coast. The ELA descends sharply from $30^{\circ}$ to $40^{\circ} \mathrm{S}$ by as much as $5000 \mathrm{~m}$.
This abrupt descent in the ELA is attributed to the prevailing westerlies south of $35^{\circ} \mathrm{S}$, which carries moisture from the Pacific Ocean.

\section{SUITABLE VARIABLES FOR DESCRIBING THE ELA}

In view of the facts presented above, it is appropriate to use at least two variables, precipitation and temperature, which represent the effects of accumulation and ablation, respectively. As will be demonstrated later, radiation is also an important factor to be considered.

ELAs are known accurately on about 100 glaciers at present where the annual mass-balance measurements have been carried out for a sufficiently long period. After some trial and error, it was found that the mean temperature of the summer months, June, July and August (December, January and February for the Southern Hemisphere) in the free atmosphere at the equivalent altitude as the ELA and the annual total precipitation at the ELA are convenient variables to characterize the ELA. Air temperature of the free atmosphere has an advantage over the screen-level air temperature, because the former is more easily accessible both in Nature and in models. The free-atmospheric temperature is calculated on the basis of data from Scherhag (1969) and NCAR World Weather Disc Records-Upper Air (TD-9648).

The annual total precipitation measured on the ELA is extremely rare. The annual total precipitation on the ELA is, therefore, approximated by the winter mass 
Table 1. Energy balance on the glacier equilibrium line during the melt period in $W^{-2}$, values in brackets are in per cent of total source or sink (numbers in the first column correspond those in Table 3)

No. Glacier Localion Allilude Surface
Lal., Long
ma.s.l.

\begin{tabular}{|c|c|c|c|c|c|c|c|c|c|c|c|}
\hline 1 & $\begin{array}{l}\text { Ward Hunt } \\
\text { Ice Shelf }\end{array}$ & $\begin{array}{l}83^{\circ} 12^{\prime} \mathrm{N} \\
74^{\circ} 00^{\prime} \mathrm{W}\end{array}$ & 15 & Snow/ice & $\begin{array}{l}60 \mathrm{~h} \text { in Jun } \\
\text { and Jul } 1960\end{array}$ & $\begin{array}{c}60 \\
(100)\end{array}$ & $\begin{array}{c}0 \\
(0)\end{array}$ & $\begin{array}{c}0 \\
(0)\end{array}$ & $\begin{array}{c}-60 \\
(-100)\end{array}$ & $\begin{array}{c}0 \\
(0)\end{array}$ & (1) \\
\hline 7 & Devon Island & & & & & & & & & & \\
\hline & ice cap & $\begin{array}{l}75^{\circ} 30^{\prime} \mathrm{N} \\
83^{\circ} 18^{\prime} \mathrm{W}\end{array}$ & 1320 & Snow & $\begin{array}{c}21 \text { May-11 Aug } \\
1962,1963\end{array}$ & $\begin{array}{l}16 \\
(62)\end{array}$ & $\begin{array}{c}10 \\
(38)\end{array}$ & $\begin{array}{c}-4 \\
(-14)\end{array}$ & $\begin{array}{l}-10 \\
(-40)\end{array}$ & $\begin{array}{l}-12 \\
(-46)\end{array}$ & $(2)$ \\
\hline 11 & $\begin{array}{l}\text { Greenland } \\
\text { ice sheet }\end{array}$ & $\begin{array}{l}69^{\circ} 41^{\prime} \mathrm{N} \\
49^{\circ} 38^{\prime} \mathrm{W}\end{array}$ & 1004 & Snow/ice & $\begin{array}{c}26 \text { May-7 Aug } \\
1959\end{array}$ & $\begin{array}{l}113 \\
(76)\end{array}$ & $\begin{array}{c}35 \\
(24)\end{array}$ & $\begin{array}{l}-25 \\
(-17)\end{array}$ & $\begin{array}{l}-111 \\
(-74)\end{array}$ & $\begin{array}{l}-13 \\
(-9)\end{array}$ & (3) \\
\hline 55 & $\begin{array}{l}\text { Vernagt- } \\
\text { ferner }\end{array}$ & $\begin{array}{l}46^{\circ} 50^{\prime} \mathrm{N} \\
10^{\circ} 45^{\prime} \mathrm{E}\end{array}$ & 2970 & Ice & $\begin{array}{c}45 \mathrm{~d} \text { in } \\
\text { Aug and Sep } \\
1950-53\end{array}$ & $\begin{array}{l}143 \\
(84)\end{array}$ & $\begin{array}{c}23 \\
(14)\end{array}$ & $\begin{array}{c}4 \\
(2)\end{array}$ & $\begin{array}{l}-170 \\
(-100)\end{array}$ & $\begin{array}{c}0 \\
(0)\end{array}$ & $(4)$ \\
\hline 56 & $\begin{array}{l}\text { Hintereis- } \\
\text { ferner }\end{array}$ & $\begin{array}{l}46^{\circ} 48^{\prime} \mathrm{N} \\
10^{\circ} 45^{\prime} \mathrm{E}\end{array}$ & 2960 & Snow/ice & $\begin{array}{c}15 \text { Jul-18 Aug } \\
1971\end{array}$ & $\begin{array}{c}66 \\
(67)\end{array}$ & $\begin{array}{c}32 \\
(33)\end{array}$ & $\begin{array}{l}-3 \\
(-4)\end{array}$ & $\begin{array}{l}-95 \\
(-96)\end{array}$ & $\begin{array}{c}0 \\
(0)\end{array}$ & (5) \\
\hline 57 & $\begin{array}{l}\text { Rhone- } \\
\text { gletscher }\end{array}$ & $\begin{array}{l}46^{\circ} 37^{\prime} \mathrm{N} \\
08^{\circ} 24^{\prime} \mathrm{E}\end{array}$ & 2820 & Snow/ice & $\begin{array}{l}1 \text { Aug-9 Sep } \\
1982\end{array}$ & $\begin{array}{c}90 \\
(53)\end{array}$ & $\begin{array}{c}81 \\
(47)\end{array}$ & $\begin{array}{l}-2 \\
(-1)\end{array}$ & $\begin{array}{l}-167 \\
(-99)\end{array}$ & $\begin{array}{c}0 \\
(0)\end{array}$ & (6) \\
\hline 63 & $\begin{array}{c}\text { No. } 1 \text { Glacier } \\
\text { Urumqi }\end{array}$ & $\begin{array}{l}\mathrm{r} \\
43^{\circ} 06^{\prime} \mathrm{N} \\
87^{\circ} 15^{\prime} \mathrm{E}\end{array}$ & 3910 & Snow/ice & $\begin{array}{c}30 \text { Aug-3 Sep } \\
1985 \\
1 \text { Jun-31 Aug } \\
1986,1987\end{array}$ & $\begin{array}{c}64 \\
(79)\end{array}$ & $\begin{array}{c}17 \\
(21)\end{array}$ & $\begin{array}{l}-15 \\
(-19)\end{array}$ & $\begin{array}{l}-66 \\
(-81)\end{array}$ & $\begin{array}{c}0 \\
(0)\end{array}$ & (7), (8) \\
\hline
\end{tabular}

\section{References}

(1) Lister (1962); (2) Holmgren (1971); (3) Ambach (1963); (4) Hoinkes (1955); (5) Tanzer (1986);

(6) Funk (1985); (7) Ohmura and Funk (1986); (8) Calanca and Heuberger (1990).

balance and the summer precipitation measured on the ELA, both of which are more often observed than the annual total precipitation. The difference between the accumulation on a glacier and the meteorologically observed precipitation will be discussed later in detail.

The radiation data, which are best suited for characterizing the glacier ELA, must be net radiation. As shown in Table 1 , this is the prime energy source for the melt. It is, however, preferable to use global radiation plus long-wave net radiation to parameterize the ELA instead of net radiation, because the observation of net radiation on an ELA for the entire melt period is extremely rare and is liable to be influenced by local conditions, such as the albedo below the net radiometer. In addition, there is another advantage in avoiding the involvement of the albedo, because the parameterization of the ELA can be more conveniently formulated by using the glacier's external factors as independent variables and the albedo should be considered as the glacier's internal characteristics, which should be found as a solution. The radiative fluxes, as well as other climatological elements which are used in this work, are summarized in Table 2 .

\section{CLIMATIC CHARACTERISTICS OF THE EQUILIBRIUM LINE}

The information on the mean equilibrium-line altitude, winter mass balance, summer precipitation, the free-atmospheric summer temperature, global radiation, global radiation plus long-wave net radiation is given in Table 3. The ELAs in this table are evaluated by massbalance measurements with stakes. The distribution of the ELAs of these 70 glaciers in the precipitationtemperature $(\mathrm{P}-\mathrm{T})$ diagram is presented in Figure 2. In general, it can be interpreted that, if the $\mathrm{P}-\mathrm{T}$ condition of a site falls in the sector within the zone of the points, such a location has a good chance of being on the ELA. If the site is not presently glacierized, it is very close to being glacierized with a slight increase in precipitation or decrease in summer temperature. If the $\mathrm{P}-\mathrm{T}$ condition falls above the zone of the points, the site is likely to be found in the accumulation area. Likewise, if the $\mathrm{P}-\mathrm{T}$ value falls below the zone of the dots, the site is either in the ablation area or unglacierized. It is assumed that there exists a function of $\mathrm{P}$ and $\mathrm{T}$ at the ELA, $f(\mathrm{P}, \mathrm{T})=0$, which satisfies the condition for creat- 
Table 2. Radiative components on or near the glacier equilibrium line

\begin{tabular}{|c|c|c|c|c|c|c|c|}
\hline \multirow[t]{2}{*}{ Glacier } & \multicolumn{2}{|c|}{ Global radiation } & \multicolumn{2}{|c|}{$\begin{array}{l}\text { Long-wave } \\
\text { net radiation }\end{array}$} & \multicolumn{2}{|c|}{$\begin{array}{l}\text { Sum of global and } \\
\text { long-wave net radiation }\end{array}$} & Sources \\
\hline & \multicolumn{2}{|c|}{$\mathrm{W} \mathrm{m}^{-2}$ (kly/3 mon.) } & \multicolumn{2}{|c|}{$\mathrm{W} \mathrm{m}{ }^{-2}$ (kly $/ 3$ mon.) } & \multicolumn{2}{|c|}{$\mathrm{W} \mathrm{m}^{-2}$ (kly/3 mon.) } & \\
\hline \multicolumn{8}{|l|}{ Ward Hunt } \\
\hline Ice Shelf & 207 & (39) & -15 & $(-3)$ & 192 & (36) & $(1),(2)$ \\
\hline White Glacier & 239 & (45) & -27 & $(-5)$ & 212 & (40) & $(3),(4),(5)$ \\
\hline Laika Glacier & 227 & (43) & -17 & $(-3)$ & 210 & (39) & (6) \\
\hline Devon Ice Cap & 270 & (51) & -69 & $(-13)$ & 201 & (38) & (7) \\
\hline $\begin{array}{l}\text { EGIG IV, } \\
\text { Greenland } \\
\text { Ram River }\end{array}$ & 212 & (40) & -42 & $(-8)$ & 170 & (32) & (8), (9), (10) \\
\hline Glacier & 260 & (49) & -43 & $(-8)$ & 217 & $(40)$ & (1), (11) \\
\hline Peyto Glacier & 244 & (46) & -37 & $(-7)$ & 207 & (39) & (12) \\
\hline Place Glacier & 242 & (46) & -32 & $(-6)$ & 210 & $(40)$ & (1), (13) \\
\hline $\begin{array}{l}\text { Sentinel Glacier } \\
\text { Nisqually }\end{array}$ & 233 & (44) & -21 & $(-4)$ & 212 & (40) & $(1),(14)$ \\
\hline Glacier & 260 & (49) & -37 & $(-7)$ & 223 & $(42)$ & $(1),(12)$ \\
\hline Vernagtferner & 245 & (46) & -19 & $(-4)$ & 226 & (43) & $(15),(16),(17)$ \\
\hline Hintereisferner & 281 & (53) & -40 & $(-8)$ & 241 & (45) & (18), (19) \\
\hline $\begin{array}{l}\text { Rhonegletscher } \\
\text { No. } 1 \text { Glacier }\end{array}$ & 292 & (55) & -53 & $(-10)$ & 239 & $(45)$ & (20) \\
\hline Urumqi & 250 & $(46)$ & -42 & $(-8)$ & 208 & (38) & $(21),(22),(23)$ \\
\hline Law Dome & 223 & (42) & -5 & $(-1)$ & 218 & (41) & $(24),(25)$ \\
\hline
\end{tabular}

Source references

(1) Canada. Department of Transport. Meteorological Branch (1970); (2) Sagar (1962); (3) Andrews (1964); (4) Havens (1964); (5) Ohmura (1981); (6) Ohmura (1977); (7) Holmgren (1971); (8) Ambach (1963); (9) Ambach (1977); (10) Marshunova and Chernigovskiy (1971); (11) Young and Stanley (1976a); (12) Young and Stanley (1976b); (13) Mokievsky-Zubok and Stanley (1976b); (14) Mokievsky-Zubok and Stanley (1976a); (15) Ambach (1955); (16) Moser and others (1986);

(17) Escher-Vetter (1985); (18) Palz and others (1979); (19) Wagner (1979); (20) Funk (1985); (21) Bai and Xie (1965); (22) Bai and others (1985); (23) Ohmura (1990); (24) Schwerdtfeger (1984); (25) Japanese Antarctic Research Expedition (1985).

ing the glacier equilibrium line. The best-fit polynomial regression curve for the 70 glaciers under consideration is $\mathrm{P}=a+b \mathrm{~T}+c \mathrm{~T}^{2}$, whereby $a=645, b=296$ and $c=9$, and $\mathrm{P}$ and $\mathrm{T}$ are in $\mathrm{mm}$ w.e. and ${ }^{\circ} \mathrm{C}$, respectively. The standard error of estimate is $200 \mathrm{~mm}$ w.e. Although the scatter of the points around the regression line is relatively narrow, it can be explained as being largely due to the different radiation condition. Global radiation alone does not explain the discrepancy amongst the dots, however, because of the often-observed negative correlation between global and net radiation for the ELA region (Ambach, 1974). The inclusion of long-wave radiation data makes it possible to understand the scatter. This result offers a possibility for parameterizing the ELA for climate models.

It is currently possible to estimate global radiation and long-wave net radiation on equilibrium lines for only 15 glaciers. The reason for the difficulty in calculating this component for other glaciers is the lack of observations on long-wave radiation. The general trend of each glacier around the regression line in Figure 2 is that for a given annual precipitation the glacier equilibrium lines under the lower summer temperature are found for glaciers with greater amounts of radiation, and vice versa. It appears that a temperature difference of $1^{\circ} \mathrm{C}$ is roughly equivalent to a $7 \mathrm{~W} \mathrm{~m}^{-2}(1.3 \mathrm{kly} / 3$ months $)$ radiation difference and $350 \mathrm{~mm}$ w.e. annual precipitation.

\section{RELATIONSHIP BETWEEN PRECIPIT- ATION AND ACCUMULATION}

Although accumulation originates primarily from precipitation, it is quantitatively different. It is, however, necessary to clarify the differences between these quantities. Of the 70 glaciers listed in Table 3, 12 glaciers are identified as suitable for a comparison of the annual precipitation and the combined amount of the winter balance and summer precipitation (Table 4). For some glaciers, the annual precipitation was measured at the mean ELA, 


\begin{tabular}{|c|c|c|c|c|c|c|c|c|c|c|c|c|}
\hline & ษัّ & 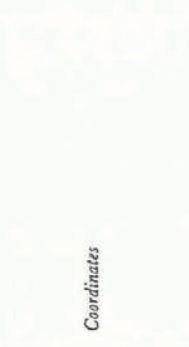 & 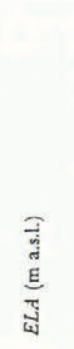 & 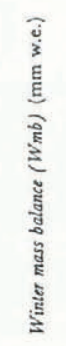 & 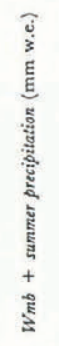 & 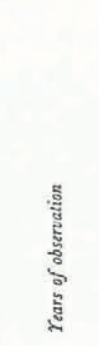 & 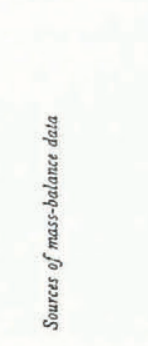 & 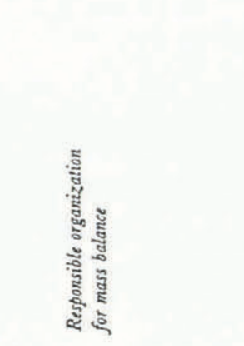 & 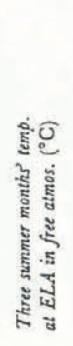 & 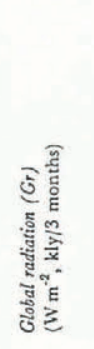 & 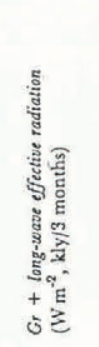 & 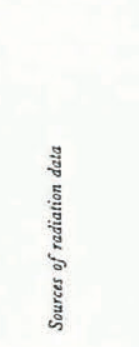 \\
\hline \multicolumn{13}{|c|}{ Canadian Arctic } \\
\hline $1 \mathrm{v}$ & Ward Hunt Ice & & & & & & & DPB & -1.0 & 207,39 & 192,36 & (1), (2) \\
\hline & & $83^{\circ} 07^{\circ} \mathrm{N}, 74^{\circ} 10^{\circ} \mathrm{W}$ & 0 & 180 & 240 & $1959-69$ & (1), (2) & Geogr. Branch & -1.1 & 240,45 & & \\
\hline & Gilman Glacier & $82^{\circ} 10^{\circ} \mathrm{N}, 71^{\circ} 15^{\circ} \mathrm{W}$ & 1250 & 110 & 170 & $51-61$ & (3), (4) & GG, ETH & -1.5 & 242,45 & & (3) \\
\hline $3 \mathrm{P}$ & Per Ardua & $81^{\circ} 27^{\circ} \mathrm{N}, 76^{\circ} 35^{\circ} \mathrm{W}$ & 1350 & 150 & 190 & $67-68$ & & McGill Univ,; GG, ETH & -1.6 & 239,45 & 212,40 & $(4),(5),(6)$ \\
\hline & White Glacier & $79^{\circ} 30^{\circ} \mathrm{N}, 90^{\circ} 58^{\circ} \mathrm{W}$ & 855 & 180 & 310 & $66-76$ & (6) & McGill Univ.; GG, ETH & -1.7 & 233,42 & & \\
\hline $5 \mathrm{~B}$ & Baby Glacier & $79^{\circ} 26 \mathrm{~N}, 90^{\circ} 58^{\mathrm{WW}}$ & 935 & 200 & 350 & $68-76$ & (7) & GG, ETH & 0.5 & 227,43 & 210,39 & (8) \\
\hline $6 \mathrm{I}$ & Laika Glacier & $75^{\circ} 53^{\circ} \mathrm{N}, 79^{\circ} 10^{\circ} \mathrm{W}$ & 355 & 410 & 590 & $72-79$ & (8) & PCSP & -0.3 & 270,51 & 201,38 & (9) \\
\hline & $\begin{array}{l}\text { Devon Island ice } \\
\text { cap, northwest }\end{array}$ & & & & & & & & & & & \\
\hline & $\begin{array}{l}\text { slope } \\
\text { Barnes Ice Cap }\end{array}$ & $\begin{array}{l}75^{\circ} 15^{\circ} \mathrm{N}, 82^{\circ} 00^{\circ} \mathrm{W} \\
70^{\circ} 10^{\circ} \mathrm{N}, 73^{\circ} 30^{\circ} \mathrm{W}\end{array}$ & $\begin{array}{r}1050 \\
810\end{array}$ & $\begin{array}{l}180 \\
440\end{array}$ & $\begin{array}{l}240 \\
590\end{array}$ & $\begin{array}{l}62-66 \\
70-76\end{array}$ & $\begin{array}{l}(9),(10) \\
(11)\end{array}$ & Glaciol. Div. & 1.9 & 202,38 & & (1) \\
\hline $9 \mathrm{I}$ & $\begin{array}{l}\text { Bannes Ice Cap } \\
\text { Decade Glacier }\end{array}$ & $69^{\circ} 39^{\circ} \mathrm{N}, 69^{\circ} 55^{\circ} \mathrm{W}$ & 1175 & 240 & 310 & $65-70$ & (5) & Glaciol. Div. & -0.3 & 196,37 & & (1) \\
\hline \multicolumn{13}{|c|}{ Greenland } \\
\hline $10 \mathrm{C}$ & Gree::land ice sheet & $76^{\circ} 30^{\circ} \mathrm{N}, 68^{\circ} 00^{\circ} \mathrm{W}$ & 650 & 410 & 700 & Stratigr. & $(12),(13)$ & CRREL & 0.6 & 238,45 & & (3) \\
\hline $11 \mathrm{c}$ & Greenland ice sheet & $69^{\circ} 30^{\circ} \mathrm{N}, 48^{\circ} 20^{\prime} \mathrm{W}$ & 1200 & 420 & 510 & $82-89$ & & GGU & 1.1 & 212,40 & 170,32 & (10), (11), (3) \\
\hline $12 \mathrm{c}$ & Qapiarfiup sermia & $65^{\circ} 36^{\circ} \mathrm{N}, 52^{\circ} 08^{\circ} \mathrm{W}$ & 790 & 1120 & 1350 & $80-85$ & $(15),(16)$ & GGU & 2.9 & 200,38 & & (12), (3) \\
\hline $13 \mathrm{c}$ & Greenland ice sheet & $64^{\circ} 30^{\circ} \mathrm{N}, 49^{\circ} 32^{2} \mathrm{~W}$ & 1310 & 590 & 990 & $81-84$ & (17) & GGU & 2.0 & 208,39 & & \\
\hline $14 \mathrm{C}$ & Greenland ice sheet & $61^{\circ} 30^{\circ} \mathrm{N}, 45^{\circ} 23^{\mathrm{WV}}$ & 1500 & 590 & 870 & $79-83$ & (17) & GGU & 2.3 & 228,43 & & (13) \\
\hline \multicolumn{13}{|c|}{ Iceland } \\
\hline $15 \mathrm{~V}$ & $\begin{array}{l}\text { Vatnajökull, south- } \\
\text { east slope }\end{array}$ & $64^{\circ} 20^{\circ} \mathrm{N}, 16^{\circ} 00^{\prime} \mathrm{W}$ & 1100 & 2000 & 2600 & Stratigr. & (18) & Univ. Iceland & 4.0 & 175,33 & & $(12),(3)$ \\
\hline \multicolumn{13}{|c|}{ Stalbard } \\
\hline 16 & Austfonna & $79^{\circ} 30^{\circ} \mathrm{N}, 25^{\circ} 15^{\circ} \mathrm{E}$ & 335 & 850 & $9: 0$ & Stratigr. & (19) & SPRI & 0.5 & 170,32 & & $(12),(3)$ \\
\hline \multicolumn{13}{|c|}{ Alaska and Cordillera } \\
\hline $17 \mathrm{C}$ & Gulkana Glacier & $63^{\circ} 18^{\prime} \mathrm{N}, 145^{\circ} 25^{\prime} \mathrm{W}$ & 1785 & 1100 & 2450 & $65-67$ & $(20),(21)$ & USGS & 3.5 & 174,33 & & (12) \\
\hline & Wolverine Glacier & $60^{\circ} 24^{\circ} \mathrm{N}, 148^{\circ} 54^{\circ} \mathrm{W}$ & 1260 & 1300 & 3600 & $65-67$ & (20), (21) & USGS & 5.6 & 174,33 & & (12) \\
\hline \multirow[t]{2}{*}{$19 \mathrm{~L}$} & Lemon Creek & $58^{\circ} 23^{\prime} \mathrm{N}, 134^{\circ} 14^{\prime} \mathrm{W}$ & 1090 & 1750 & 2850 & & & & & 195,37 & & \\
\hline & $\begin{array}{l}\text { Glacier } \\
\text { Alexander Glacier }\end{array}$ & $\begin{array}{l}58^{\circ} 23^{\circ} \mathrm{N}, 134^{\circ} 14^{\circ} \mathrm{W} \\
57^{\circ} 06^{\circ} \mathrm{N}, 130^{\circ} 49^{\circ} \mathrm{W}\end{array}$ & $\begin{array}{l}1090 \\
1750\end{array}$ & $\begin{array}{l}1750 \\
1800\end{array}$ & $\begin{array}{l}2850 \\
2050\end{array}$ & $\begin{array}{l}56-58 \\
73-84\end{array}$ & $\begin{array}{l}(22) \\
(11)\end{array}$ & $\begin{array}{l}\text { Michigan State Univ. } \\
\text { Glaciol. Div. }\end{array}$ & $\begin{array}{l}7.0 \\
3.7\end{array}$ & $\begin{array}{l}195,37 \\
195,37\end{array}$ & & (1) \\
\hline & $\begin{array}{l}\text { Alexander Glacier } \\
\text { Yuri Glacier }\end{array}$ & $56^{\circ} 58^{\circ} \mathrm{N}, 130^{2} 42^{\circ} \mathrm{W}$ & 1829 & 1600 & 1850 & $77-84$ & $\begin{array}{l}(11) \\
\text { (11) }\end{array}$ & $\begin{array}{l}\text { Glaciol. Div. } \\
\text { Glaciol. Div. }\end{array}$ & & $\begin{array}{l}195,37 \\
195,37\end{array}$ & & (1) \\
\hline $\begin{array}{ll}21 & Y \\
22 & A\end{array}$ & $\begin{array}{l}\text { Yuri Glacier } \\
\text { Andrei Glacier }\end{array}$ & $56^{\circ} 57^{\circ} \mathrm{N}, 130^{\circ} 59^{\circ} \mathrm{W}$ & 1510 & 1900 & 2150 & $77-84$ & $\begin{array}{l}\text { (11) } \\
\text { (11) }\end{array}$ & $\begin{array}{l}\text { Glaciol. Div. } \\
\text { Glaciol. Div. }\end{array}$ & $\begin{array}{l}3.3 \\
5.1\end{array}$ & $\begin{array}{l}195,37 \\
195,37\end{array}$ & & (1) \\
\hline $23 \mathrm{~F}$ & Ram River Glacier & $51^{\circ} 51^{\prime} \mathrm{N}, 116^{\circ} 11^{\prime} \mathrm{w}$ & 2860 & 1000 & 1180 & $65-74$ & $\begin{array}{l}(11) \\
(5),(10)\end{array}$ & Glaciol. Div. & 4.7 & 260,49 & 217,40 & $\begin{array}{l}\text { (1) } \\
\text { (1), (14) }\end{array}$ \\
\hline $24 \mathrm{~F}$ & Peyto Glacier & $51^{\circ} 40^{\circ} \mathrm{N}, 116^{\circ} 33^{\circ} \mathrm{W}$ & 2670 & 1400 & 1600 & $65-80$ & $(5),(10),(23)$ & Glaciol. Div. & 6.1 & 244,46 & 207,39 & $(15)$ \\
\hline $25 \mathrm{~B}$ & Bench Glacier & $51^{\circ} 27^{\prime} \mathrm{N}, 124^{\circ} 56^{\prime} \mathrm{W}$ & 1860 & 2000 & 2300 & $80-84$ & (24) & Glaciol. Div. & 6.4 & 223,42 & & (1) \\
\hline & Tiedemann Glacier & $51^{\circ} 20^{\circ} \mathrm{N}, 125^{\circ} 00^{\circ} \mathrm{W}$ & 1890 & 2300 & 2550 & $80-84$ & (24) & Glaciol. Div. & 6.2 & 223,42 & & (1) \\
\hline 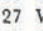 & Woolsey Glacier & $51^{\circ} 01^{\prime} \mathrm{N}, 118^{\circ} 12^{\prime} \mathrm{W}$ & 2270 & 2700 & 3100 & $65-74$ & (5), (10) & Glaciol. Div. & 8.9 & 260,49 & & (1) \\
\hline $28 \mathrm{~s}$ & Sykora Glacier & $30^{\circ} 53^{\circ} \mathrm{N}, 123^{\circ} 34^{\mathrm{W}} \mathrm{W}$ & 2200 & 1900 & 2050 & $76-84$ & (11) & Glaciol. Div. & 4.5 & 240,45 & & (1) \\
\hline $29 \mathrm{~B}$ & Bridge Glacier & $50^{\circ} 49^{\circ} \mathrm{N}, 123^{\circ} 33^{\prime} \mathrm{W}$ & 2200 & 1950 & 2100 & $76-84$ & (11i) & Glaciol. Div. & 4.5 & 240,45 & & (1) \\
\hline 302 & Zabisha Glacier & $50^{\circ} 48^{\circ} \mathrm{N}, 123^{\circ} 25^{\circ} \mathrm{W}$ & 2280 & 1730 & 1900 & $76-84$ & (11) & Glaciol. Div. & 4.1 & 240,45 & & \\
\hline $31 \mathrm{I}$ & Place Glacier & $50^{\circ} 26^{\circ} \mathrm{N}, 122^{\circ} 36^{\circ} \mathrm{W}$ & 2155 & 2100 & 2240 & $64-74$ & (5), (10) & Glaciol. Div. & 6.8 & 242,46 & 210,40 & (1), (16) \\
\hline $32 \mathrm{~F}$ & Helm Glacier & $49^{\circ} 58^{\circ} \mathrm{N}, 123^{\circ} 00^{\circ} \mathrm{W}$ & 2090 & 2100 & 2350 & $76-84$ & (ii) & Glaciol. Div. & 5.0 & 240,45 & & \\
\hline $33 \mathrm{~s}$ & Sentinel Glacier & $49^{\circ} 54^{\prime} \mathrm{N}, 122^{\circ} 59^{\circ} \mathrm{W}$ & 1805 & 3500 & 3750 & $65-75$ & $(5),(10)$ & Glaciol. Div. & 8.7 & 233,44 & 212,40 & (1), (17) \\
\hline $34 \mathrm{Sc}$ & $\begin{array}{l}\text { South Cascade } \\
\text { Glacier }\end{array}$ & $48^{\circ} 45^{\circ} \mathrm{N}, 121^{\circ} 03^{\circ} \mathrm{W}$ & 1965 & 2650 & 2900 & $64-80$ & (5), (23) & & & 260,49 & & (12), (1) \\
\hline $35 \mathrm{~s}$ & $\begin{array}{l}\text { Glacier } \\
\text { Nisqually Glacier }\end{array}$ & $46^{\circ} 08^{\circ} \mathrm{N}, 121^{\circ} 44^{\circ} \mathrm{W}$ & 2080 & 4900 & 3150 & $67-68$ & $\begin{array}{l}(3),(23) \\
(5)\end{array}$ & $\begin{array}{l}\text { USGS } \\
\text { USGS }\end{array}$ & $\begin{array}{l}0.9 \\
9.5\end{array}$ & $\begin{array}{l}260,49 \\
260,49\end{array}$ & 233,42 & (1), (17) \\
\hline \multicolumn{13}{|c|}{ Scandinavia } \\
\hline 361 & Blaisen & $68^{\circ} 20^{\circ} \mathrm{N}, 17^{\circ} 31^{\circ} \mathrm{E}$ & 1100 & 1700 & 1900 & $64-68$ & (5) & NWREB & 4.6 & 180,34 & & (12) \\
\hline & Storsteinfjellbreen & $68^{\circ} 13^{\circ} \mathrm{N}, 17^{\circ} 55^{\prime} \mathrm{E}$ & 1375 & 1350 & 1600 & $64-68$ & (5) & NWREB & 3.1 & 180,34 & & (12) \\
\hline 38 & Cainhavarre & $68^{\circ} 06^{\circ} \mathrm{N}, 18^{\circ} 00^{\circ} \mathrm{E}$ & 1405 & 1400 & 1600 & $64-68$ & (5) & NWREB & 2.9 & 180,34 & & (12) \\
\hline $39 s$ & Storglaciären & $67^{\circ} 54^{\circ} \mathrm{N}, 18^{\circ} 34^{\circ} \mathrm{E}$ & 1486 & 1520 & 1720 & $59-86$ & (5) & Univ. Stockholm & 3.8 & 180,34 & & (12) \\
\hline $40^{\circ}$ & Trollbergdalsbreen & $66^{\circ} 43^{\prime} \mathrm{N}, 14^{\circ} 27^{\prime} \mathrm{E}$ & 1065 & 2950 & 3200 & $70-75$ & (10) & NWREB & 5.6 & 180,33 & & (12) \\
\hline 41 & Engabreen & $66^{\circ} 39^{\circ} \mathrm{N}, 13^{\circ} 51^{\prime} \mathrm{E}$ & 1085 & 2700 & 3050 & $69-80$ & (5), (10), (23) & NWREB & 6.3 & 180,33 & & (12) \\
\hline 42 & Högtuvbreen & $66^{\circ} 27^{\prime} \mathrm{N}, 13^{\circ} 39^{\prime} \mathrm{E}$ & 845 & 3450 & 3850 & $70-77$ & $(10),(23)$ & NWREB & 7.7 & 180,33 & & (12) \\
\hline 43 & Alfotbreen & $61^{\circ} 45^{\circ} \mathrm{N}, \quad 5^{\circ} 39^{\circ} \mathrm{E}$ & 1175 & 3450 & 3770 & $64-80$ & (5), (10), (23) & NWREB & 6.8 & 180,34 & & (12) \\
\hline 44 & Nigardsbreen & $61^{\circ} 43^{\prime} \mathrm{N}, 7^{\circ} 08^{\prime} \mathrm{E}$ & 1545 & 2000 & 2300 & $64-80$ & (3), (10), (23) & NWREB & 4.9 & 180,34 & & (12) \\
\hline 45 & Grasubreen & $61^{\circ} 39^{\circ} \mathrm{N}, \quad 8^{\circ} 36^{\prime} \mathrm{E}$ & 2145 & 750 & 1000 & $64-80$ & (5), (10), (23) & NWREB & 1.9 & 180,34 & & (12) \\
\hline 461 & Hellstungubreen & $61^{\circ} 34^{\prime} \mathrm{N}, \quad 8^{\circ} 26^{\prime} \mathrm{E}$ & 1925 & 1200 & 1450 & $64-80$ & $(5),(10),(23)$ & NWREB & 2.9 & 180,34 & & (12) \\
\hline 47 & Tunsbergdalsbreen & $61^{\circ} 36^{\circ} \mathrm{N}, \quad 7^{\circ} 03^{\circ} \mathrm{E}$ & 1465 & 2150 & 2400 & $65-70$ & (5) & NWREB & 5.2 & 180,34 & & (12) \\
\hline 48 & Austre Memurubre & $61^{\circ} 33^{\prime} \mathrm{N}, \quad 8^{\circ} 30^{\circ} \mathrm{E}$ & 2045 & 1250 & 1500 & $67-70$ & (5) & NWREB & 2.1 & 180,34 & & (12) \\
\hline 49 & Vestre Memurubre & $61^{\circ} 32 \mathrm{~N}, \quad 8^{\circ} 27^{\circ} \mathrm{E}$ & 2010 & 1350 & 1600 & $67-70$ & (5) & NWREB & 2.3 & 180,34 & & (12) \\
\hline 50 & Vesledalsbreen & $61^{\circ} 05^{\circ} \mathrm{N}, \quad 7^{\circ} 16^{\prime} \mathrm{E}$ & 1390 & 2300 & 2550 & $66-72$ & (5), (10) & NWREB & 5.9 & 180,34 & & (12) \\
\hline 51 & Hardangerjökulen & $60^{\circ} 33^{\circ} \mathrm{N}, \quad 7^{\circ} 22^{\mathrm{E}}$ & 1665 & 1850 & 2200 & $64-75$ & (5), (10) & NWREB & 3.5 & 182,34 & & (12), (18) \\
\hline 52 & Folgefonni-East & $60^{\circ} 09 \mathrm{~N}, \quad 6^{\circ} 29^{\circ} \mathrm{E}$ & 1435 & 2700 & 3150 & $64-68$ & (5) & NWREB & 5.7 & 186,35 & & (18) \\
\hline 53 & Folgefonni-West & $60^{\circ} 09^{\circ} \mathrm{N}, \quad 6^{\circ} 29^{\prime} \mathrm{E}$ & 1475 & 2600 & 3050 & $64-68$ & (5) & NWREB & 5.4 & 186,35 & & (18) \\
\hline & Bondhusbreen & $60^{\circ} 02^{\circ} \mathrm{N}, \quad 6^{\circ} 20^{\circ} \mathrm{E}$ & 1525 & 2400 & 2850 & $76-80$ & (23) & NWREB & 5.1 & 186,36 & & (18) \\
\hline
\end{tabular}




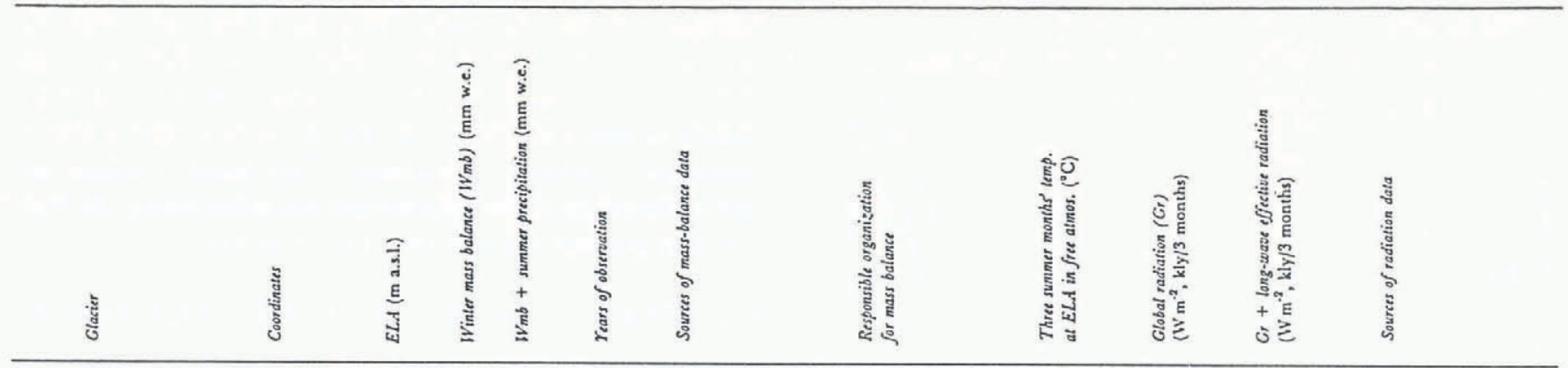

\begin{tabular}{|c|c|c|c|c|c|c|c|c|c|c|c|c|}
\hline \multicolumn{13}{|c|}{ Alps } \\
\hline 55 & Vernagterner & $46^{\circ} 52^{\circ} \mathrm{N}, 10^{\circ} 49^{\prime} \mathrm{E}$ & 3085 & 1000 & 1550 & $64-85$ & (25) & KGBAW & 2.0 & 245,46 & 226,43 & $(19<(20),(21)$ \\
\hline 56 & Hintereisferner & $46^{\circ} 48^{\mathrm{N}}, 10^{\circ} 46^{\circ} \mathrm{E}$ & 2960 & 1500 & 2100 & $66-78$ & $(26),(27)$ & DMG, UI & 2.0 & 281,53 & 241,45 & (22), (23), (18) \\
\hline 37 & Rhonegletscher & $46^{\circ} 37^{\circ} \mathrm{N}, \quad 8^{\circ} 24^{\circ} \mathrm{E}$ & 2950 & 1950 & 2230 & $79-82$ & (28) & GG, ETH & 2.2 & 292,55 & 239,45 & (24) \\
\hline 58 & Careser & $46^{\circ} 27^{\circ}, 10^{\circ} 41^{\circ} \mathrm{E}$ & 3105 & 1150 & 1450 & $66-79$ & (5), (10), (23) & IGUP & 1.6 & 255,48 & & (12) \\
\hline 59 & Griesgletscher & $46^{\circ} 26^{\circ} \mathrm{N}, \quad 8^{\circ} 20^{\circ} \mathrm{E}$ & 2870 & 1050 & 1800 & $64-85$ & (29) & VAW, ETH & 2.7 & 250,47 & & (18) \\
\hline \multirow[t]{2}{*}{60} & Marmolada & $44^{\circ} 07^{\circ} \mathrm{N}, \quad 7^{\circ} 23^{\circ} \mathrm{E}$ & 2740 & 1050 & 1250 & $64-66$ & (5) & IGUP & 4.3 & 275,52 & & $(12),(18)$ \\
\hline & \multicolumn{12}{|l|}{ Caucasus } \\
\hline \multirow[t]{2}{*}{61} & Dzankuat & $43^{\circ} 12^{\circ} \mathrm{N}, 42^{\circ} 44^{\circ} \mathrm{E}$ & 3210 & 2250 & 2800 & $67-80$ & (23) & GFMSU & 4.1 & 265,30 & & (12) \\
\hline & \multicolumn{12}{|l|}{ Tianshan } \\
\hline \multirow{4}{*}{$\begin{array}{l}62 \\
63\end{array}$} & Tsentrainyy & & & & & & & & & & & \\
\hline & $\begin{array}{l}\text { Tuyuksu } \\
\text { No. } 1 \text { Glacier }\end{array}$ & $43^{\circ} 10^{\circ} \mathrm{N}, 77^{\circ} 00^{\circ} \mathrm{E}$ & 3835 & 1000 & 1400 & $64-80$ & $(5),(10),(23)$ & GS, ANKSSR & 3.6 & 280,53 & & (12) \\
\hline & Urumqi & $43^{\circ} 07^{\prime} \mathrm{N}, 86^{\circ}+9^{\prime} \mathrm{E}$ & 4050 & 350 & 400 & $59-74$ & (10) & LIGG, AS & -0.2 & 250,45 & 208,38 & $(25),(26)$ \\
\hline & \multicolumn{12}{|l|}{ Himalaya } \\
\hline \multirow[t]{2}{*}{64} & Rikha Samba & & & & & & & & & & & \\
\hline & Glacier & $29^{\circ} 00^{\circ} \mathrm{N}, 83^{\circ} 30^{\circ} \mathrm{E}$ & 3650 & 20 & 270 & 74 & (30) & WRI, UN & -1.3 & 223,42 & & (27) \\
\hline 65 & Gyajo Glacier & $27^{\circ} 54^{\circ} \mathrm{N}, 86^{\circ}+1^{\prime} \mathrm{E}$ & 5370 & 50 & 900 & 76 & (31) & WRI, UN & 0.3 & 207,39 & & (28) \\
\hline \multirow[t]{2}{*}{66} & EB050 (E09) & $27^{\circ} 38^{\circ} \mathrm{N}, 86^{\circ}+6^{\circ} \mathrm{E}$ & 5270 & 30 & 1300 & 76 & $(31),(32)$ & WRI, UN & 0.9 & 212,40 & & (28) \\
\hline & \multicolumn{12}{|l|}{ Southern Hemisphere } \\
\hline 67 & Tasman Glacier & $43^{\circ} 30^{\circ} \mathrm{S}, 170^{\circ} 20^{\circ} \mathrm{E}$ & 1900 & 2500 & 3250 & $66-71$ & (5) & GS, MIW & 7.7 & 205,39 & & (12) \\
\hline \multirow{3}{*}{69} & Hodges Glacier & $54^{\circ} 17^{\circ} \mathrm{S}, 36^{\circ} 30^{\circ} \mathrm{W}$ & 435 & 1480 & 1850 & $5 !-60$ & (33) & UA & 1.9 & 215,40 & & (29) \\
\hline & Glacier, Deception & & & & & & & & & & & \\
\hline & Island & $63^{\circ} 00^{\circ} \mathrm{S}, 60^{\circ} 40^{\circ} \mathrm{W}$ & 325 & 600 & 780 & $68-71$ & (5) & IPS, OSU & 0.0 & 185,35 & & (12) \\
\hline 70 & Law Dome & $66^{\circ} 00^{\circ} \mathrm{S}, 112^{\circ} 00^{\circ} \mathrm{E}$ & 150 & 330 & 360 & Stratigr. & (34) & ANARE & -2.6 & 223,42 & 218,41 & (30), (31) \\
\hline
\end{tabular}

Responsible organization for mass balance

ANARE Australian National Antarctic Research Expeditions

CRREL U.S. Army Cold Regions Research and Engineering Laboratory

DMG, UI Department of Meteorology and Geophysics, University of Innsbruck

DRB Defense Research Board, Canada

Geogr. Branch Geographical Branch, Department of Transport, Canaci

GFMSU Geographical Faculty, Moscow State University

GG, ETH Department of Geography, Eidgenössische Technische Hochschule, Zürich

GGU Geological Survey of Greenland Denmark

Glaciol. Div. Glaciological Division, Inland Waters Branch, Department of Energy, Mines and Resources, Canada

GS, ANKSSR Geographical Section, Academy of Sciences, Kazakhstan Soviet Socialist Republic

IGUP Institute of Geography, University of Padua

KGBAW Commission of Glaciology, Bavarian Academy of Sciences

IPS, OSU Institute of Polar Studies, Ohio State University

GS, MW Glaciology Section, Ministry of Works, New Zealand

LIGG, AS L.anzhou Institute of Glaciology and Geocryology, Academia Sinica, China

NWREB Norwegian Water Resources and Electricity Board

PCSP Polar Continental Shelf Project, Department of Energy, Mines and Resources,

SPRI Scott Polar Research Institute, Cambridge, England

UA University of East Anglia, England

USGS U.S. Geological Survey

VAW, ETH Versuchsanstalt für Wasserbau, Hydrologie und Glazialogie, Eidgenössische

WRI, UN Water Resources Institute, University of Nagova

such as Rhonegletscher and Griesgletscher. For other glaciers, such as Laika Glacier and Law Dome, meteorological stations were located very near (within $300 \mathrm{~m}$ altitude) the ELA. For glaciers of the other group, such as White Glacier and No. 1 Urumqi glacier, the meteorological stations were closely located but with a much larger altitude difference. In these regions, however, the altitudinal dependency of precipitation is well estab-
Sources of mass-balance data

(1) Hattersley-Smith and Serson (1970); (2) Koerner (1979); (3) Sagar (1964); (4) Arnold (1968); Kasser (1973); (6) Weiss (1984); Alean (1977); (8) Kraus (1983); (9) Koerner (1966); (10) Muller (1977); (11) Mokievsky-Zubok and others (1985); (12) Schytt (1955); (13) Benson (1962); (14) Thomsen (personal communication); (15) Olesen (1986); (16) Weidick and Thomsen (1986); (17)
Thent (197) (11) Thomsen (personal communication); (15) Olesen (1986); (16) Weidick and Thomsen (1986); (17)
Braithwaite (personal communication); (18) Björnsson (personal communication); (19) Dowdeswell Braithwaite (personal communication); (18) Björnsson (personal communication); (19) Dowdeswell
(personal comunication); (20) Meier and others (1971); (21) Tangborn and others (1977); (22) (personal comunication); (20) Meier and others (1971); (21) Tangborn and others (1977); (22)
Marcus (1964); (23) Haeberli (1985); (24) Mokievsky-Zubok (1985); (25) Moser and others (1986); Marcus (1964); (23) Haeberli (1985); (24) Mokievsky-Zubok (1985); (25) Moser and others (1986);
(26) Hoinkes (1970); (27) Kuhn (1981); (28) Funk (1985); (29) Funk and Aellen (unpublished); (30) (26) Hoinkes (1970); (27) Kuhn (1981); (28) Funk (1985); (29) Funk and Aellen (unpublished); (30)
Fujii and others (1976); (31) Ageta and Satow (1978); (32) Yasunari and Inoue (1978); (33) Timmis (1986); (34) Xie (1984).

Sources of radiation date

(1) Canada. Department of Transport. Meteorological Branch (1970); (2) Sagar (1962); (3 Marshunova and Chernigovskiy (1971); (4) Andrews (1964); (5) Havens (1964); (6) Ohmur (1982); (7) Ohmura (1981); (8) Ohmura (1977); (9) Holmgren (1971); (10) Ambach (1963); (11) Ambach (1977); (12) Budyko (1963); (13) Braithwaite and Olesen (1984); (14) Young and Stanle (1976a); (15) Young and Stanley (1976b); (16) Mokievsky-Zubok and Stanley (1976b); (17) Mokievsky-Zubok and Stanley (1976a); (18) Palz and others (1979); (19) Ambach (1955); (20 Moser and others (1986); (21) Escher-Vetter (1985); (22) Kuhn (1981); (23) Wagner (1979); (24) Funk (1985); (25) Wang and Zhang (1985); (26) Bai and others (1985); (27) Higuchi (1977); (28) Mani (1980); (29) Shanklin (1981); (30) Japanesc Antaretic Research Expedition (1985); Schwerdt feger (1984)

lished, so that it was possible to correct the annual total meteorological precipitation to the ELA. On all these glaciers, the summer precipitation and the winter glacier mass balance were measured at altitudes very close to the ELA or right on the long-term ELA (White Glacier, Rhonegletscher and No. 1 Urumqi glacier).

The comparison between the annual precipitation and the combined winter balance and summer precipitation 


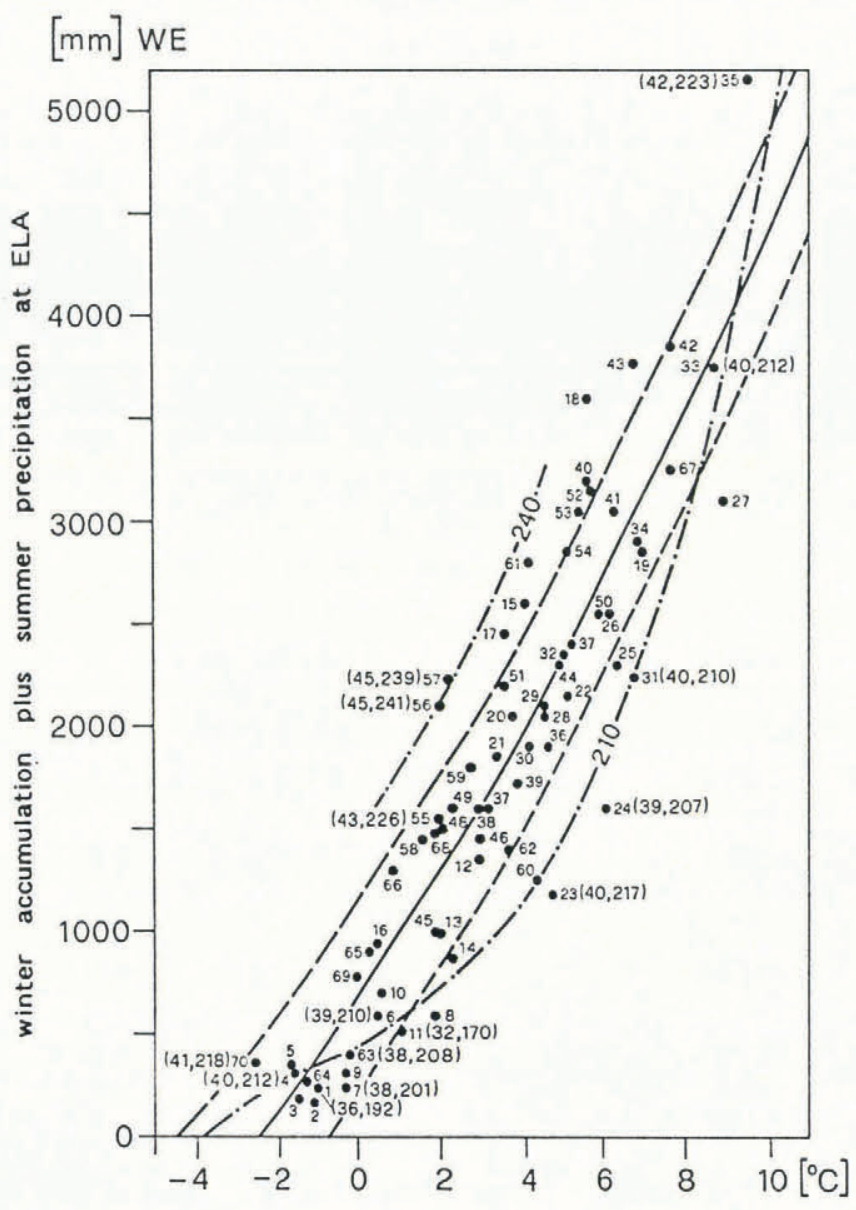

mean summer temperature in free atmosphere at ELA

Fig. 2. Annual total precipitation (or winter mass balance plus summer precipitation) and the mean free-atmospheric temperature observed at the ELAs for 70 glaciers. The numbers indicate the glaciers listed in Table 3. The solid line and the broken lines indicate the square regression line and the standard deviation, respectively. The numbers in brackets are global plus long-wave net radiation for the summer 3 months, June, July and August (December, January and February for the Southern Hemisphere), expressed in kly/3 months and $\mathrm{W} \mathrm{m}^{-2}$, respectively. The dotted and dashed lines indicate the best-fit curves for the glaciers with summer radiation 240 and $210 \mathrm{~W} \mathrm{~m}^{-2}$.

shows that they are very close to each other. This is especially true for White Glacier, Rhonegletscher and Griesgletscher, for which meteorological and glaciological observations are considered to be of a very high quality. On average, the metorologically measured annual precipitation is slightly smaller than the winter balance plus summer precipitation. This is partly due to the known underestimation of precipitation gauges, particularly of solid precipitation (Sevruk, 1983). For some glaciers, however, the winter mass balance is clearly larger than the meteorologically measured precipitation, and the accumulation through snow drift may be an important accumulation mechanism for such glaciers (Laika
Glacier, Woolsey Glacier, Hintereisferner and Tsentralnyy Tuyuksu). The most important conclusion of this comparison is that the winter mass balance (accumulation) comes very close to the meteorological precipitation on a number of glaciers. This point justifies the approximation of the annual precipitation using the winter balance and the summer precipitation.

\section{CLIMATIC CHANGE AND THE ELA SHIFT}

The sensitivity of the ELA with respect to the change in climatic elements is examined. The shift of the ELA was investigated by Kuhn (1981) from the energy-balance viewpoint. In the present work, the statistical trend in the relationship developed in the previous section is used.

(a)

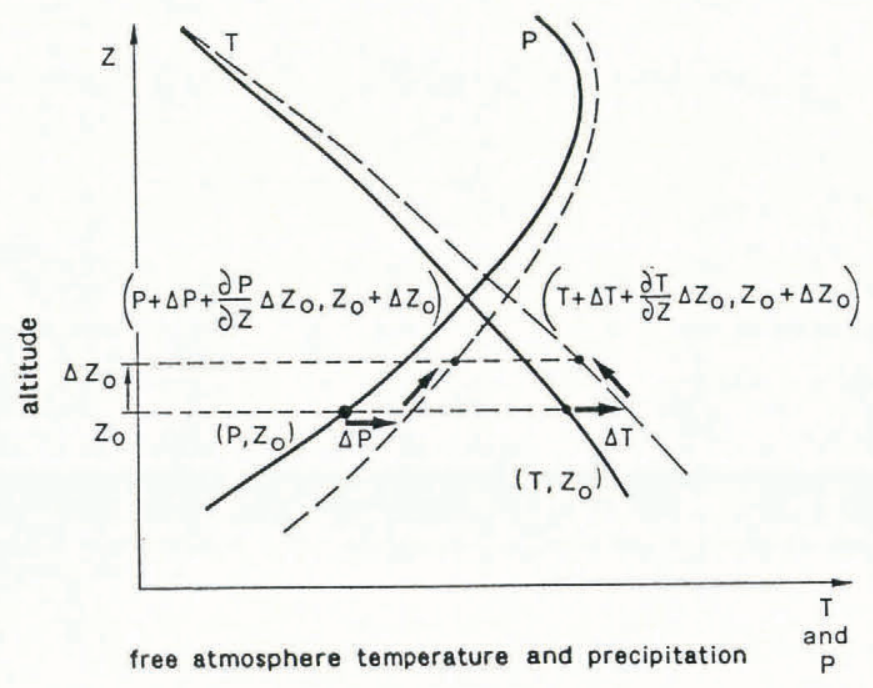

(b)

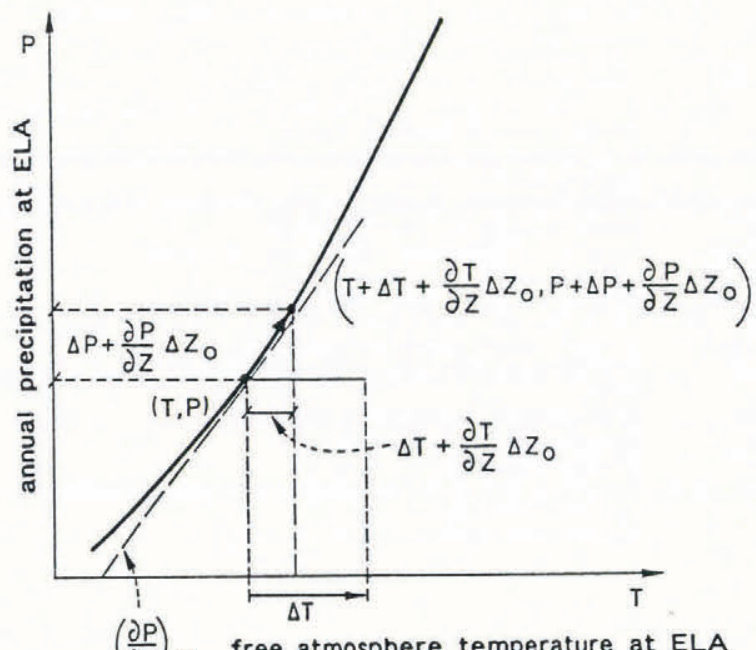

$\left(\frac{\partial P}{\partial T}\right)_{Z_{0}}$ free atmosphere temperature at ELA

Fig. 3.a. Vertical distributions of annual precipitation and summer free atmospheric temperature, before (solid line) and after (broken line) the climatic change which resulted in the shift of the equilibrium line by $\Delta z_{0}$. b. Dislocation of the equilibrium-line precipitation and temperature on the $P-T$ diagram as presented in Figure 2. 
Table 4. Comparison of precipitation and accumulation on glaciers (mmw.e.) on ELA

\begin{tabular}{|c|c|c|c|c|c|c|}
\hline $\begin{array}{l}\text { Annu } \\
\text { meteor } \\
\text { precip }\end{array}$ & $\begin{array}{l}\text { cal total } \\
\text { rological } \\
\text { pitation }\end{array}$ & $\begin{array}{c}\text { Years } \\
\text { of observation }\end{array}$ & $\begin{array}{c}\text { Source of meteor- } \\
\text { ological data }\end{array}$ & $\begin{array}{l}\text { Glaciological winter } \\
\text { balance plus meteor- } \\
\text { ological summer }\end{array}$ & $\begin{array}{c}\text { Years of } \\
\text { observations }\end{array}$ & $\begin{array}{c}\text { Source of } \\
\text { glaciological } \\
\text { data }\end{array}$ \\
\hline White Glacier & 300 & 1969-72 & (1) & 310 & 1969-72 & (17) \\
\hline Laika Glacier & 340 & $1972-74$ & (2) & 590 & $1972-74$ & (18) \\
\hline Qamanarssup & & & & & & \\
\hline $\begin{array}{l}\text { sermia } \\
\text { Woolsey Glacier }\end{array}$ & $\begin{array}{r}720 \\
2500\end{array}$ & $\begin{array}{l}1980-84 \\
1951-80\end{array}$ & $\begin{array}{l}(3),(4),(5) \\
(6)\end{array}$ & $\begin{array}{r}990 \\
3100\end{array}$ & $\begin{array}{l}1980-84 \\
1965-74\end{array}$ & $\begin{array}{l}(3),(4),(5) \\
(11),(19)\end{array}$ \\
\hline Hintereisferner & 1500 & $\begin{array}{l}1957-59 \\
1974-78\end{array}$ & $(7),(8),(9)$ & 2100 & $\begin{array}{l}1957-59 \\
1974-78\end{array}$ & $\begin{array}{l}(7) \\
(8)\end{array}$ \\
\hline $\begin{array}{l}\text { Rhonegletscher } \\
\text { Tsentralnyy }\end{array}$ & 2400 & 1918-22 & (10) & 2250 & 1979-82 & (20) \\
\hline $\begin{array}{l}\text { Tuyuksu } \\
\text { No. } 1 \text { Glacier }\end{array}$ & 960 & $1959-70$ & (11) & 1390 & $1964-70$ & (11) \\
\hline Urumqi & 650 & 1958-78 & (12) & 570 & 1959-66 & $(21)$ \\
\hline Hodges Glacier & 1450 & $1951-60$ & (13) & 1850 & $\begin{array}{l}1957-58 \\
1972-77\end{array}$ & (22) \\
\hline $\begin{array}{l}\text { Glacier, Decep- } \\
\text { tion Island }\end{array}$ & 560 & $1944-67$ & (14) & 780 & $1968-71$ & - \\
\hline Law Dome & 280 & $1958-60$ & (15) & 320 & $\begin{array}{l}\text { Not spec- } \\
\text { ified }\end{array}$ & (23) \\
\hline Griesgletscher & 1710 & $1964-85$ & (16) & 1800 & $1964-85$ & (16) \\
\hline
\end{tabular}

\section{Source references}

(1) Ohmura (1980); (2) Ohmura (1976); (3) Braithwaite (1987); (4) Braithwaite (1989); (5) Braithwaite (personal communication); (6) Atmospheric Environment Service (1982); (7) Hoinkes and Lang (1962); (8) Kuhn (1981); (9) Kuhn and others (1982); (10) Schweiz Bundesamt für Hydrologie (unpublished); (11) Kasser (1973); (12) Yang and others (1988); (13) WMO (1971); (14) British Meteorological Office (1978); (15) Schwerdtfeger (1984); (16) Funk and Aellen (unpublished); (17) Weiss (1984); (18) Blatter and Kappenberger (1988); (19) Müller (1977); (20) Funk (1985); (21) Zhang (1981); (22) Timmis (1986); (23) Xie (1984).

For the sake of simplicity, only the variation of temperature and precipitation is considered.

In Figure 3a, annual precipitation $P$ and summer temperature in a free atmosphere $T$ at the original ELA denoted by $z_{0}$ are indicated as solid lines which represent the present climate. The change in the climate for the altitude $z_{0}$ is represented by $\Delta P$ and $\Delta T$. As the result of the climatic change, the position of the ELA is shifted to $z_{0}+\Delta z_{0}$ where the new precipitation and temperature are approximated by $P+\Delta P+\partial P / \partial z \Delta z_{0}$ and $T+\Delta T+\partial T / \partial z \Delta z_{0}$, respectively. The new precipitation and temperature should be on the solid line of the ELA in Figure 3b. The linear approximation of the new location of the ELA in the P-T diagram is presented in Figure 3b. The relationship between the new temperature and precipitation should be

$$
\Delta P+\frac{\partial P}{\partial z} \Delta z_{0}=\left(\frac{\partial P}{\partial T}\right)_{z_{0}}\left\{\Delta T+\frac{\partial T}{\partial z} \Delta z_{0}\right\}
$$

where $(\partial P / \partial T)_{z_{0}}$ is the gradient of the function
$f(P, T)=0$ in Figure 2, and $(\partial P / \partial T)_{z_{0}}=b+2 c T$. Rearranging Equation (1) for $\Delta z_{0}$

$$
\Delta z_{0}=\frac{\Delta T-\Delta P\left(\frac{\partial P}{\partial T}\right)_{z_{0}}^{-1}}{\frac{\partial P}{\partial z}\left(\frac{\partial P}{\partial T}\right)_{z_{0}}^{-1}-\frac{\partial T}{\partial z}} .
$$

Equation (2) represents several important features of the climate/glacier relationship. The vertical shift of the ELA is linear with the change in temperature. The ELA shift is also linear with the decrease in precipitation, although the effectiveness of the precipitation change is not so large because $(\partial P / \partial T)_{z_{0}}^{-1}=-2.5$ to $-3.3 \times 10^{-3} \mathrm{~K} \mathrm{~mm}^{-1}$. This statement is justified, as $\partial P / \partial T, \partial P / \partial z$ and $\partial T / \partial z$ are almost constant and therefore independent of changes in temperature and precipitation. This means that a change in precipitation of $300-400 \mathrm{~mm}$ w.e. corresponds to only $1^{\circ} \mathrm{C}$ temperature change. 
For the same change of temperature and precipitation, the glaciers in a region of large lapse rate $\Gamma=-\partial T / \partial z$ react less sensitively in comparison to those of small lapse rate. Since the regions of larger lapse rate are associated with a continental climate, the glaciers in arid environments must behave insensitively towards climatic changes, and vice versa.

\section{ELA AND MASS-BALANCE SENSITIVITY}

Each glacier possesses a different mass-balance sensitivity with respect to the shift of the ELA. The relationship between the annual mass balance and the ELA makes it possible to translate the shift of the ELA into the change in mass balance and holds important information concerning the effect of climatic changes on the glacierization. The ELA sensitivity of the mass balance is defined as the partial differential of mean annual specific balance by the ELA, $\partial \bar{b} / \partial$ ELA and calculated as the gradient of the $\bar{b}$-ELA diagram. The mass-balance sensitivity of the ELA shift is calculated for 36 glaciers, for which longterm records of the mass balance and good topographic maps are available. Considering that the sensitivity can be parameterized by the annual specific turnover of the mass $(\tau)$ and the surface gradient $(\alpha)$ of the glacier, Figure 4 is made by taking $\tau / \alpha$ as an independent variable, whereby $\tau=(\bar{c}+|\bar{a}|) / 2, \bar{c}$ and $\bar{a}$ being the mean specific accumulation and ablation, respectively.

The explanation as to how the parameter $\tau / \alpha$ is suited for expressing the mass-balance sensitivity $\partial \bar{b} / \partial$ ELA is given below. We consider a simplified two-dimensional glacier of a unit length (projected on the horizontal sur-

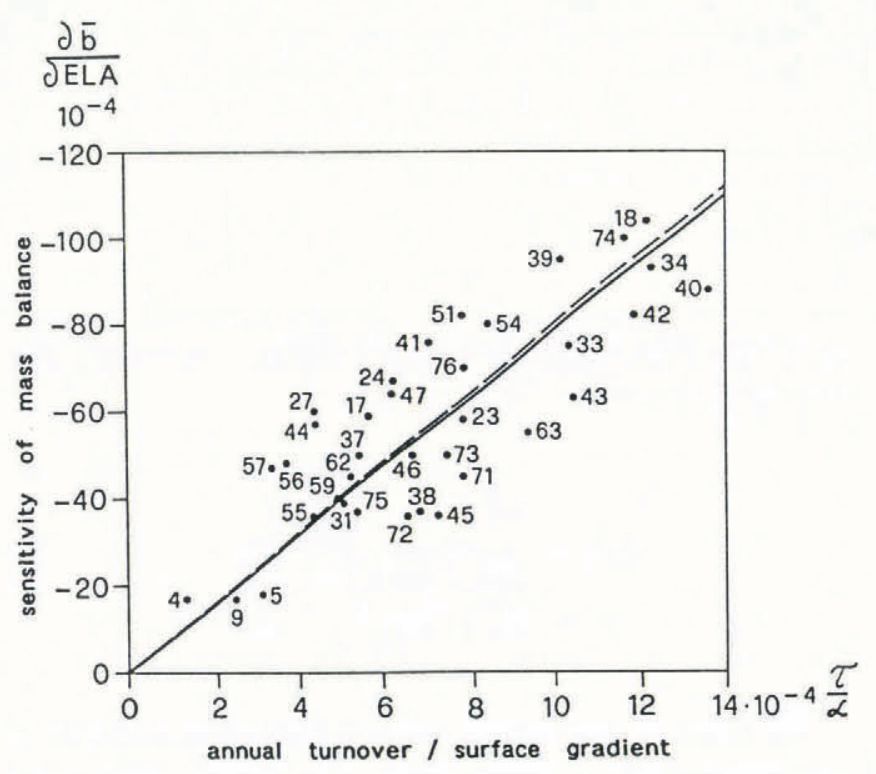

Fig. 4. The mass-balance sensitivity of the ELA shift expressed as a function of the annual mass turnover and the surface gradient of glaciers. The solid and broken lines indicate the linear regression line and the theoretical prediction, respectively. Numbers correspond to those in Table 3, except for 71 (Blue Glacier), 72 (Sonnblick Kees), 73 (Silvretta Gletscher), 74 (Kesselwandferner), 75 (Limmerngletscher) and 76 (Langtalerferner).

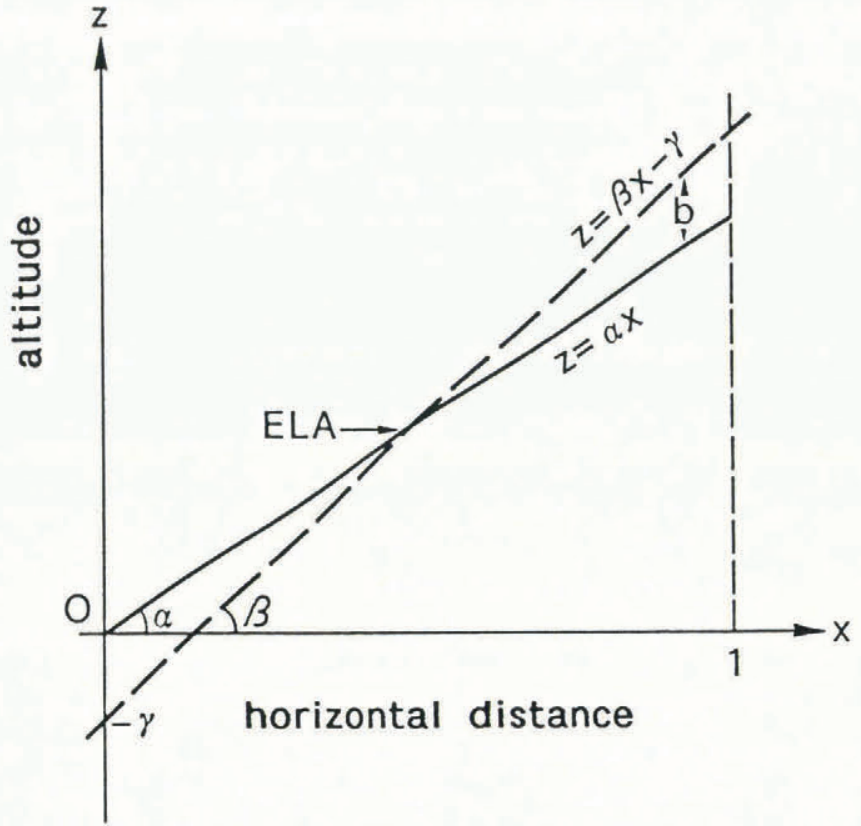

Fig. 5. Linear expressions of the glacier surface and the change of the surface due only to annual mass balance. The solid and broken lines indicate the glacier surface with gradient $\alpha$ and the surface as a result of the mass balance $b$, but before the surface has been adjusted by dynamics. $X$ and $Z$ indicate the horizontal distance and vertical height, where the horizontally projected glacier length is defined as unity.

face), with a constant surface gradient $\alpha$ and density, extending from the origin of the coordinate system as illustrated in Figure 5. The surface change which is expected due to the mass balance of one budget year is also expressed by a linear equation $z=\beta x-\gamma$. If more complicated expressions for the surface altitude and the mass balance are desired, non-linear curves can be used. The $x$ and $z$ coordinates of the equilibrium line are found to be $(\gamma /(\beta-\alpha), \alpha \gamma /(\beta-\alpha))$. The $z$-coordinate difference between the two lines is what we define as the annual mass balance in ice equivalent, therefore

$$
b=(\beta-\alpha) x-\gamma .
$$

Then, the mass-balance gradient of this glacier is

$$
\frac{\partial b}{\partial z}=\frac{\partial b}{\partial x} \frac{\partial x}{\partial z}=\frac{\beta-\alpha}{\alpha} .
$$

The total mass balance can be expressed as

$$
B=\int_{0}^{1} b \mathrm{~d} x=(\beta-\alpha) \int_{0}^{1} x \mathrm{~d} x-\gamma \int_{0}^{1} \mathrm{~d} x .
$$

Consequently,

$$
B=\frac{\beta-\alpha}{2}-\gamma=\bar{b}
$$

Equation (6) is justified, because the glacier has a unit 
length. The glacier in steady state has $B=0$, therefore

$$
\gamma=\frac{\beta-\alpha}{2}
$$

Then, the mass-balance sensitivity is

$$
\frac{\partial \bar{b}}{\partial \mathrm{ELA}}=\frac{\partial \bar{b}}{\partial z_{0}}=\frac{\partial \bar{b}}{\partial \gamma} \frac{\partial \gamma}{\partial z_{0}}=\frac{\alpha-\beta}{\alpha}
$$

where $z_{0}$ is the altitude of the equilibrium-line ELA. Therefore,

$$
\frac{\partial \bar{b}}{\partial \mathrm{ELA}}=-\frac{\partial b}{\partial z} .
$$

Insofar as the linear approximation of the glacier surface and the mass balance are concerned, the mass-balance sensitivity becomes the same quantity as the negative of the mass-balance gradient.

However, since the mass-balance gradient is in reality variable depending on the altitude, it is desirable to replace it with a more stable quantity which characterizes the entire glacier. We use the concept of the annual specific mass turn-over of a glacier $\tau$, defined earlier, which is the mean rate of mass inflow or outflow with respect to the unit surface area of the glacier.

For the glacier under consideration:

$$
\begin{aligned}
\tau=\frac{1}{2} & \left\{\int_{0}^{\gamma /(\beta-\alpha)}[\gamma-(\beta-\alpha) x] \mathrm{d} x\right. \\
& \left.+\int_{\gamma /(\beta-\alpha)}^{1}[(\beta-\alpha) x-\gamma] \mathrm{d} x\right\} \\
= & \frac{1}{2}\left(\frac{\gamma^{2}}{\beta-\alpha}+\frac{\beta-\alpha}{2}-\gamma\right) .
\end{aligned}
$$

For glaciers with near steady state, that is Equation (7) holds, and

$$
\tau \approx \frac{\beta-\alpha}{8} \text { or } \beta-\alpha \approx 8 \tau \text {. }
$$

Therefore,

$$
\frac{\partial \bar{b}}{\partial \mathrm{ELA}}=-8 \frac{\tau}{\alpha}
$$

Equation (12) is applicable whether $\bar{b}$ and $\tau$ are water or ice equivalent, so long as the same unit is used for both. The straight solid line in Figure 4 expresses this theoretically expected relationship, while the broken line is the statistically calculated regression line for the points. The gradient of the regression line is -7.85 and very close to the theoretical prediction of -8 . The figure demonstrates that the wide range in the variety of the ELA effect on the mean annual specific mass balance can be expressed as a function of the annual mean turnover and surface gradient, both of which are relatively easy to obtain or estimate. One of the main advantages for using the turnover instead of mass balance as a variable is that the turnover is much less variable than mass balance, owing to the complementary relationship between the absolute values of ablation and accumulation. To demonstrate this point, standard deviations of annual specific turnover and annual mean specific mass balance are compared for White Glacier, Ram River Glacier and Kesselwandferner, which represent glaciers of very small, medium and very large mass-balance sensitivity of the ELA, respectively (Table 5). These glaciers also fit very well the theoretical expectation of the relationship of the ELA mass-balance sensitivity with $\tau / \alpha$. This relationship makes it possible to estimate the massbalance change of a glacier as a result of climatic changes, given the ELA shift, mean turnover and geometry of the glacier.

\section{CONCLUSIONS}

The climate prevailing at the equilibrium lines is identified as a function of annual total precipitation and summer temperature in the free atmosphere. Refinement of the relationship is possible by introducing global and long-wave net radiation. The equivalent values for temperature, precipitation and radiation at the glacier equilibrium lines are approximately $1^{\circ} \mathrm{C}, 350 \mathrm{~mm}$ w.e. and $7 \mathrm{~W} \mathrm{~m}^{-2}$, respectively. Assuming this relationship holds, the effect of a climatic change on the shift of the equilibrium line, and further, the effect of the shift of the equilibrium line on the change in the mean specific mass balance, are evaluated. The sensitivity of the mean specific mass balance is found to be proportional to

Table 5. Comparison of the standard deviations of the annual specific turn-over and annual mean specific mass balance for selected glaciers (in $\mathrm{mm}$ w.e.)

Glaciers

Standard deviations

Period of observation

Source

Turn-over Mass balance

$\begin{array}{lrlll}\text { White Glacier } & 58 & 266 & 1959 / 60-1978 / 79 & (1) \\ \text { Ram River Glacier } & 155 & 540 & 1965 / 66-1974 / 75 & (2),(3) \\ \text { Kesselwandferner } & 106 & 295 & 1965 / 66-1980 / 81 & (2),(3),(4)\end{array}$

(1) Weiss (1984); (2) Kasser (1973); (3) Müller (1977); (4) Haeberli (1985). 
the annual mass turnover and reciprocally proportional to the surface gradient. The proportionality constant is -8 when the longitudinal length of a glacier is taken as a unit for lengths such as altitude and mass balance. The present work offers a possibility of predicting the mass-balance change of a glacier resulting from a climatic change.

\section{ACKNOWLEDGEMENTS}

The authors thank the following individuals and organizations for unpublished scientific data: H. Björnsson for the ELA on Vatnajökull, R. J. Braithwaite and H. H. Thomsen for the ELAs on the west slope of the Greenland ice sheet, J. Dowdeswell for the ELA on Austfonna, Lanzhou Institute of Glaciology and Geocryology for climatological data for the Tien Shan and Altay Mountains, and the Danish Meteorological Institute for climatological data on Greenland. The present work has been possible, due partly to the high-quality massbalance data from Norway. We should like to thank Professor G. Østrem for the organization which made this task possible. Our own mass-balance measurements in the Canadian Arctic were generously supported by the Polar Continental Shelf Project, DEMR, Canada. A part of the present work was financed by the Research Fund of the Eidgenössische Technische Hochschule (ETH) No. 0330.060.34/4 and the Swiss National Science Foundation grant No. 2.307-0.86, No. 20-25271.88 and No. 20-29826.90 for re-evaluation of the global heat balance.

\section{REFERENCES}

Ageta, Y. and K. Satow. 1978. Study of the mass balance of small glaciers in Khumbu Himal during the summer monsoon season. Seppyo, 40, Special Issue, 4-11.

Ahlmann, H.W. 1948. Glaciological research on the North Atlantic coasts. London, Royal Geographical Society. (Research Series 1.)

Alean, J. 1977. Zum Massenhaushalt des Baby Glaciers auf der Axel Heiberg Insel in der kanadischen Hocharktis (1959/60 bis 1975/76). (Diplomarbeit, Eidgenössische Technische Hochschule, Zürich.)

Ambach, W. 1955. Über den nächtlichen Wärmeumsatz der gefrorenen Gletscheroberfläche. Arch. Meteorol. Geophys. Bioklimatol., Ser. A, 8(4), 411-426.

Ambach, W. 1963. Untersuchungen zum Energieumsatz in der Ablationszone des grönländischen Inlandeises (Camp IV - EGIG, $69^{\circ} 40^{\prime} 05^{\prime \prime} \mathrm{N}, 49^{\circ} 37^{\prime} 58^{\prime \prime} \mathrm{W}$ ). Medd. Grønl., 174(4).

Ambach, W. 1974. The influence of cloudiness on the net radiation balance of a snow surface with high albedo. J. Glaciol., 13(67), 73-84.

Ambach, W. 1977. Untersuchungen zum Energieumsatz in der Ablationszone des grönländischen Inlandeises. Nachtrag. Medd. Grønl., 187(5).

Ames, A. 1985. Estudio de mediciónes glaciologicas efectuadas en la Cordillera Blanca per Electropern S.A.: variación y balance de masas de los glaciares y su contribución en el caudal de las cuencas. Grenoble, Laboratoire de Glaciologie et de Géophysique de l'Environnement. (Publication 457.)
Andrews, R.H. 1964. Meteorology and heat balance of the ablation area, White Glacier, Canadian Arctic Archipelago - summer 1960 (Lower Ice Station: $\left.79^{\circ} 26^{\prime} \mathrm{N}, 90^{\circ} 39^{\prime} \mathrm{W}, 208 \mathrm{~m}\right)$. Axel Heiberg Isl. Res. Rep., McGill Univ., Meteorol. 1.

Arnold, K. C. 1968. Determination of changes of surface height, 1957-1967, of Gilman Glacier, northern Ellesmere Island, Canada. (M.Sc. thesis, McGill University.)

Atmospheric Environment Service. 1982. Canadian climate normals 1951-1980. Vol. 3. Precipitation. Toronto, Environment Canada. Atmospheric Environment Service.

Bai, Z. and W. Xie. 1965. Heat balance on the melting surface during the ablation period on Glacier No. 1 in the headwater of Urumqi river, Tianshan. In Glaciological and hydrological research in the headwater of Urumqi river. Beijing, Science Press, 75-82.

Bai, Z., L. Ding and T. Yuz. 1985. Preliminary results of the investigation on the relations among the radiation - and heat balance, glaciers and climate in the headwater of Urumqi river, Tianshan. Annual Report of Work at Tianshan Glaciological Station, 2, 199-241.

Benson, C. S. 1962. Stratigraphic studies in the snow and firn of the Greenland ice sheet. SIPRE Res. Rep. 70.

Blatter, H. and G. Kappenberger. 1988. Mass balance and thermal regime of Laika Ice Cap, Coburg Island, N.W.T., Canada. J. Glaciol., 34(116), 102-110.

Braithwaite, R. J. 1980. Regional modelling of ablation in West Greenland. Grønl. Geol. Undersøgelse. Rapp. 98.

Braithwaite, R. J. 1987. The last full summer of glacierclimate investigations at Qamanârssûp sermia, West Greenland. Grønl. Geol. Undersøgelse. Rapp. 135, 95-99.

Braithwaite, R.J. 1989. Glacier-climate studies 19871988 at Qamanârssûp sermia, West Greenland. Grønl. Geol. Undersøgelse. Rapp. 145, 56-58.

Braithwaite, R. J. and O.B. Olesen. 1985. Ice ablation in West Greenland in relation to air temperature and global radiation. Z. Gletscherkd. Glazialgeol., 20, 1984, 155-168.

British Meteorological Office. 1978. Tables of temperature, relative humidity and precipitation for the world. Part II. London, Her Majesty's Stationery Office.

Budyko, M.I., ed. 1963. Atlas teplovogo balansa zemnogo shara [Atlas of the heat balance of the Earth]. Moscow, Akademiya Nauk, SSSR.

Calanca, P. -L. and R. Heuberger. 1990. Energy balance. Zürcher Geogr. Schr. 38, 60-73.

Canada. Department of Transport. Meteorological Branch. 1970. Atlas of climatic maps. Toronto, Department of Transport. Meteorological Branch.

Escher-Vetter, H. 1985. Energy balance calculations for the ablation period 1982 at Vernagtferner, Oetztal Alps. Ann. Glaciol., 6, 158-160.

Fujii, Y., M. Nakawo and M. L. Shrestha. 1976. Mass balance studies of the glaciers in Hidden Valley, Mukut Himal. Seppyo, 38, Special Issue, 17-21. 
Funk, M. 1985. Räumliche Verteilung der Massenbilanz auf dem Rhonegletscher und ihre Beziehung zu Klimaelementen. Zürcher Geogr. Schr. 24.

Funk, M. and M. Aellen. Unpublished. Bericht No. 5026 über die Hydrologie und Glaziologie des Griesseegebietes im Jahr 1984/85 mit Uerbersicht der Hauptergebnisse seit 1961/62 im Auftrag der Kraftweke Aegina AG, Ulrichen und Zürich, Juli 1986.

Haeberli, W., comp. 1985. Fluctuations of glaciers 1975-1980. Vol. IV. Paris, International Association of Hydrological Sciences/UNESCO.

Hattersley-Smith, G. and H. Serson. 1970. Mass balance of the Ward Hunt ice rise and Ice Shelf: 10 year record. J. Glaciol., 9(56), 247-252.

Havens, J. M. 1964. Meteorology and heat balance on the accumulation area, McGill Ice Cap, Canadian Arctic Archipelago - summer 1960 (Upper Ice Station 1: $\left.79^{\circ} 41^{\prime} \mathrm{N} 90^{\circ} 27^{\prime} \mathrm{W}, 1530 \mathrm{~m}\right)$. Axel Heiberg Isl. Res. Rep., McGill Univ., Meteorol. 2.

Higuchi, K. 1977. Effect of nocturnal precipitation on the mass balance of the Rikha Samba Glacier, Hidden Valley, Nepal. Seppyo, 39, Special Issue, 43-49.

Hoinkes, H. 1955. Measurements of ablation and heat balance on Alpine glaciers. J. Glaciol., 2(17), 497501.

Hoinkes, H. 1970. Methoden und Möglichkeiten von Massenhaushaltsstudien auf Gletschern. Z. Gletscherkd. Glazialgeol., 6(1-2), 38-90.

Hoinkes, H. and H. Lang. 1962. Winterschneedecke und Gebietsniederschlag 1957/58 und 1958/59 im Bereich des Hintereis- und Kesselwandferners (Ötztaler Alpen). Arch. Meteorol. Geophys. Bioklimatol., Ser. B, 11(4), 424-446.

Holmgren, B. 1971. Climate and energy exchange on a sub-polar ice cap in summer. Arctic Institute of North America Devon Island Expedition 1961-1963. Part E. Radiation climate. Uppsala Universitet. Meteorologiska Institutionen. (Meddelande 111.)

Japanese Antarctic Research Expedition. 1985. Science in Antarctica. Vol. 9. Data compilation. Tokyo, National Institute of Polar Research.

Johnson, A. M. 1976. The climate of Peru, Bolivia and Ecuador. In Schwerdtfeger, W., ed. World survey of climatology. Vol. 12. Amsterdam, Elsevier, 147-218.

Jordan, E. 1984. Die Vergletscherung des Cotopaxi Ecuador. Z. Gletscherkd. Glazialgeol., 19(1), 1983, 73-102.

Källén, E., C. Crafoord and M. Ghil. 1979. Free oscillation in a climate model with ice-sheet dynamics. $J$. Atmos. Sci., 36(12), 2292-2303.

Kasser, P., comp. 1973. Fluctuations of glaciers 19651970. Paris, International Association of Hydrological Sciences/UNESCO.

Koerner, R. M. 1966. Accumulation on the Devon Island ice cap, Northwest Territories, Canada. J. Glaciol., 6(45), 383-392.

Koerner, R. M. 1979. Accumulation, ablation; and oxygen isotope variations on the Queen Elizabeth Islands ice caps, Canada. J. Glaciol., 22(86), 25-41.

Kraus, P. 1983. Glacier inventory of southeast Ellesmere
Island, N.W.T., Canada and its application for estimating the annual run-off. Zürcher Geogr. Schr. 9.

Kuhn, M. 1981. Climate and glaciers. International Association of Hydrological Sciences Publication 131 (Symposium at Canberra 1979 - Sea Level, Ice, and Climatic Change), 3-20.

Kuhn, M., U. Nickus and F. Pellet. 1982. Die Niederschlagsverhältnisse im inneren Ötztal. Kurzfassungen der Vorträge. 17, ITAM, Berchtesgaden, 21.-25. Sept. 1982, 235-237.

Lister, H. 1962. Heat and mass balance at the surface of the Ward Hunt Ice Shelf, 1960. Montreal, etc., Arctic Institute of North America. (Research Paper 19.)

Loewe, F. 1971. Considerations on the origin of the Quaternary ice sheet of North America. Arct. Alp. Res., 3(4), 331-344.

Mani, A. 1980. Handbook of solar radiation data for India. New Delhi, etc., Allied Publishers.

Marcus, M. G. 1964. Climate-glacier studies in the Juneau Ice Field region, Alaska. Chicago, University of Chicago Press. (Department of Geography Research Paper 88.)

Marshunova, M.S. and N. T. Chernigovskiy. 1971. Radiatsionnyy rezhim zarubezhmoy Arktiki [Radiation regime of the foreign Arctic]. Leningrad, Gidrometeorologicheskoye Izdatel'stvo.

Meier, M. F., W. V. Tangborn, L. R. Mayo and A. Post. 1971. Combined ice and water balances of Gulkana and Wolverine glaciers, Alaska and South Cascade Glacier, Washington, 1965 and 1966 hydrologic years. U.S. Geol. Surv. Prof. Pap. 725-A.

Miller, A. 1976. The climate of Chile. In Schwerdtfeger, W., ed. World survey of climatology. Vol. 12. Amsterdam, Elsevier, 113-145.

Mokievsky-Zubok, O. and A.D. Stanley. 1976a. Canadian glaciers in the International Hydrological Decade Program, 1965-1974. No. 1. Sentinel Glacier, British Columbia - summary of measurements. Ottawa, Inland Waters Directorate. Water Resources Branch. (Scientific Series 68.)

Mokievsky-Zubok, O. and A.D. Stanley. 1976b. Canadian glaciers in the International Hydrological Decade Program, 1965-1974. No. 2. Place Glacier, British Columbia — summary of measurements. Ottawa, Inland Waters Directorate. Water Resources Branch. (Scientific Series 69.)

Mokievsky-Zubok, O., C.S. L. Oinmanney and J. Power. 1985. NHRI glacier mass balance 1964-1984 (Cordillera and Arctic). Ottawa, Environment Canada. National Hydrology Research Institute. Surface Water Division. Glacier Section.

Moser, H., H. Escher-Vetter, H. Oerter, O. Reinwarth and D. Zunke. 1986. Abfluss in und von Gletschern. Munich, Gesellschaft für Strahlen- und Umweltforschung. (GSF-Bericht 41/86.)

Müller, F., comp. 1977. Fluctuations of glaciers 1970 1975. (Vol. III.) Paris, International Association of Hydrological Sciences/UNESCO.

Nogami, M. 1976. Altitude of the modern snow line in the Andes. Tokyo, Tokyo Metropolitan University, 71-86. (Geographical Report 11.) 
Oerlemans, J. and C. J. van der Veen. 1984. Ice sheets and climate. Dordrecht, etc., D. Reidel Publishing Company.

Ohmura, A. 1976. The climate of North Water 1972-75. In Müller, F., ed. North Water Project. Progress report 1 October 1974 to 30 September 1975. Zürich, ETH; Montreal, McGill University, 14-54.

Ohmura, A. 1977. Radiation and heat balance on the surface of North Water. In Müller, F., ed. North Water Project. Progress report 1 October 1975 to 30 September 1976. Zürich, ETH; Montreal, McGill University, 41-54.

Ohmura, A. 1980. The re-evaluation of the radiation budget and energy balance for the surface of North Water. In Müller, F., ed. North Water Project. Progress report VI. 1 April 1978 to 31 December 1979. Zürich, ETH; Montreal, McGill University, 2-6.

Ohmura, A. 1981. Climate and energy balance on Arctic tundra, Axel Heiberg Island, Canadian Arctic Archipelago, spring and summer 1969, 1970 and 1972. Zürcher Geogr. Schr. 3.

Ohmura, A. 1982. Climate and energy balance on the Arctic tundra. J. Climatol., 2, 65-84.

Ohmura, A. 1987. New temperature distribution maps for Greenland. Z. Gletscherkd. Glazialgeol., 23(1), $1-45$.

Ohmura, A. 1990. On the existence of Glacier No. 1, Urumqi River. Zürcher Geogr. Schr. 38.

Ohmura, A. and M. Funk. 1986. Report on energy balance measurements on Glacier No. 1 in Tianshan (August-September 1985). Zürich, Eidgenössische Technische Hochschule. (Berichte und Skripten 24.)

Ohmura, A., P. Kasser and M. Funk. 1986. Parameterization of glacierization for a climate model. Proceedings of the ISLSCP Conference, Roma, Italy, 2-6 Dec. 1985. ESA SP-248, 113-123.

Olesen, O. B. 1986. Fourth year of glaciological field work at Tasersiaq and Qapiarfiup sermia, West Greenland. Grønl. Geol. Undersøgelse. Rapp. 130, 121-126.

Palz, W., F. Kasten and H. J. Golchert, eds. 1979. European solar radiation atlas. Vol. 1. Global radiations on horizontal surfaces. Dortmund, W. Grösschen Verlag.

Prohaska, F. 1976. The climate of Argentina, Paraguay and Uruguay. In Schwerdtfeger, W., ed. World survey of climatology. Vol. 12. Amsterdam, Elsevier, 13-72.

Sagar, R.B. 1962. Meteorological and glaciological studies, Ice Rise Station, Ward Hunt Island, May to September 1960. Montreal, Arctic Institute of North America. (Research Paper 24.)

Sagar, R. B. 1964. Meteorological and glaciological observations on the Gilman Glacier, northern Ellesmere Island, 1961. Geogr. Bull., 22, 13-56.

Scherhag, R. 1969. Klimatische Karten der Nordhemisphäre. Freie Universität Berlin. Institut für Meteorologie und Geophysik. Meteorologische Abhandlungen, 100(1).

Schweiz. Bundesamt für Hydrologie. Unpublished. Internal memorandum with data on the precipitation measured by totalizers in the area of the Rhone
Glacier in 1919-1924. Federal Bureau of Hydrology, Berne, 1977.

Schwerdtfeger, W. 1984. Weather and climate of the Antarctic. Amsterdam, Elsevier. (Developments in Atmospheric Science 15.)

Schytt, V. 1955. Glaciological investigations in the Thule Ramp area. SIPRE Rep. 28.

Sevruk, B. 1983. Correction of measured precipitation in the Alps using the water equivalent of new snow. Nord. Hydrol., 14(2), 49-58.

Shanklin, J.D. 1981. Measurements of solar and terrestrial radiation at King Edward Point, South Georgia, 1973-81. Cambridge, British Antarctic Survey.

Shi, Y., ed. 1988. Maps of snow, ice and frozen ground in China. Lanzhou, Lanzhou Institute of Glaciology and Geocryology.

Tangborn, W. V., L. R. Mayo, D. R. Scully and R. M. Krimmel. 1977. Combined ice and water balances of Maclure Glacier, California, South Cascade Glacier, Washington, and Wolverine and Gulkan glaciers, Alaska, 1967 Hydrological Year. U.S. Geol. Surv. Prof. Pap. 725-B.

Tanzer, G. 1986. Berechnung des Wärmehaushalts an der Gleichgewichtslinie des Hintereisferner. (Diplomarbeit, Universität Innsbruck.)

Timmis, R.J. 1986. Glacier changes in South Georgia and their relationship to climatic trends. (Ph.D. thesis, University of East Anglia.)

U.S. Department of Commerce. 1982. World weather records 1961-1970. Vol. 3. West Indies, South and Central America. Ashville, AL, National Climatic Center.

Wagner, H. P. 1979. Strahlungshaushaltsuntersuchungen an einem Ostalpengletscher während der Hauptablationsperiode. Teil II. Langwellige Strahlung und Strahlungsbilanz. Arch. Meteorol. Geophys. Bioklimatol., Ser. B, 28, 41-62.

Wang, D. and P. Zhang. 1985. On the valley climate of Urumqi River in the Tianshan Mountains. J. Glaciol. Geocryol., 7(3), 239-248.

Weidick, A. and H.H. Thomsen. 1986. A decade of glacier investigations for utilisation of Greenland hydropower. Grønl. Geol. Undersøgelse. Rapp. 128, $157-169$.

Weiss, J. 1984. Massenhaushalt eines Gletschers - Fragen an glaziologische Untersuchungen und die Resultate der Messreihe am White Glacier in der kanadischen Arktis. (Diplomarbeit, Eidgenössische Technische Hochschule, Zürich.)

Wernstedt, F. L. 1985. World climatic data. University Park, PA, Pennsylvania State University.

WMO. 1971. Climatological normals (CLINO) for CLIMAT and CLIMAT SHIP stations for the period 1931-1960. Geneva, World Meteorological Organization. (WMO/OMM-117.)

Xiao, S. 1981. On the relation of the atmospheric water vapour and precipitation to the distribution of modern mountain glaciers in China. J. Glaciol. Cryopedol., 3(1), 45-52.

Xie, Z. 1984. Studies of ice formation and ice fabric on 
the Law Dome, Antarctica. J. Glaciol. Cryopedol., 6(1), 1-23.

Yang, D., T. Jiang, Y. Zhang and E. Kang. 1988. Analysis and correction of errors in precipitation measurement at the head of Urumqi river, Tianshan. J. Glaciol. Geocryol., 10(4), 399-407.

Yasunari, T. and J. Inoue. 1978. Characteristics of monsoonal precipitation around peaks and ridges in Shorong and Khumbu Himal. Seppyo, 40, Special Issue, 26-32.

Young, G.J. and A.D. Stanley. 1976a. Canadian glaciers in the International Hydrological Decade Program, 1965-1974. No. 3. Ram River Glacier, Alberta - summary of measurements. Ottawa, Inland Waters Directorate. Water Resources Branch. (Scientific Series 70.)
Young, G. J. and A. D. Stanley. 1976b. Canadian glaciers in the International Hydrological Decade Program, 1965-1974. No. 4. Peyto Glacier, Alberta - summary of measurements. Ottawa, Inland Waters Directorate. Water Resources Branch. (Scientific Series 71.)

Zhang, J. 1981. Mass balance studies on the No. 1 Glacier of Urumqi River in Tianshan. J. Glaciol. Cryopedol., 3(2), 32-40.

The accuracy of references in the text and in this list is the responsibility of the authors, to whom queries should be addressed. 